\title{
Interaction of plasma cloud with external electric field in lower ionosphere
}

\author{
Y. S. Dimant and M. M. Oppenheim \\ Boston University, Boston, MA, USA \\ Received: 7 August 2009 - Revised: 11 January 2010 - Accepted: 3 March 2010 - Published: 11 March 2010
}

\begin{abstract}
In the auroral lower-E and upper-D region of the ionosphere, plasma clouds, such as sporadic-E layers and meteor plasma trails, occur daily. Large-scale electric fields, created by the magnetospheric dynamo, will polarize these highly conducting clouds, redistributing the electrostatic potential and generating anisotropic currents both within and around the cloud. Using a simplified model of the cloud and the background ionosphere, we develop the first selfconsistent three-dimensional analytical theory of these phenomena. For dense clouds, this theory predicts highly amplified electric fields around the cloud, along with strong currents collected from the ionosphere and circulated through the cloud. This has implications for the generation of plasma instabilities, electron heating, and global MHD modeling of magnetosphere-ionosphere coupling via modifications of conductances induced by sporadic-E clouds.
\end{abstract}

Keywords. Electromagnetics (Plasmas) - Ionosphere (Electric fields and currents) - Space plasma physics (Electrostatic structures)

\section{Introduction}

At lower-E/upper-D ionospheric altitudes, roughly between 80 and $120 \mathrm{~km}$, high density meteor plasma trails and sporadic-E layers develop within a lower density background plasma. While the former are persistent, highly elongated, and relatively short-lived structures, the latter, much rarer events, are pancake-like clouds that span tens to hundreds of kilometers in horizontal direction and live for several hours or longer. For a review of sporadic-E layer observations and theory, see Whitehead (1970, 1989); Mathews (1998); Kirkwood and Nilsson (2000). These enhanced density regions

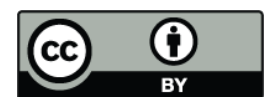

Correspondence to: Y. S. Dimant (dimant@bu.edu) coexist with a macroscopic electric field sustained by external dynamos (Kelley, 1989). In addition, neutral winds with a component perpendicular to the magnetic field produce an effective external electric field in the neutral frame of reference. Such external fields polarize the highly conducting cloud, redistribute the electrostatic potential within it and in the background ionosphere, and form a large-scale current system. This can be important for generation of plasma instabilities, electron heating, electrodynamic coupling between the ionospheric $\mathrm{E}$ and $\mathrm{F}$ regions, and, through modified ionospheric conductance, even for global ionospheremagnetosphere coupling.

Bristow and Watkins (1994) performed global 3-D simulations of sporadic-E formation and dynamics, but their coarse mesh did not allow 3-D electrodynamics. Haldoupis et al. (1996) suggested a mechanism of a sporadic-E cloud polarization by an external electric field in order to explain type 1 radar echoes from the midlatitude E-region ionosphere. In their semi-quantitative analysis, they used a slab model of the sporadic-E cloud, infinite in the north-south direction. Tsunoda (1998) and Maruyama et al. (2000) applied a similar approach to explain the generation of quasi-periodic echoes (Yamamoto et al., 1991; Ogawa et al., 1995; Pan and Tsunoda, 1998).

Shalimov et al. (1998) and Shalimov and Haldoupis (2005) applied ideas similar to those from the seminal papers by Farley $(1959,1960)$ by including ionospheric current closure and using a more realistic 3-D slab model. However, their theory relied on approximate scaling arguments rather than on a rigorous solution of the underlying differential equations. That is why their model describes no spatial distributions of fields and currents. Furthermore, Shalimov et al. (1998) and Shalimov and Haldoupis (2005) used artificial equation splitting which is invalid and they ignored vertical slab polarization, as we discuss in Sect. 4.

Hysell and Burcham (2000); Hysell et al. (2002); Yokoyama et al. (2003); Cosgrove and Tsunoda (2003) and

Published by Copernicus Publications on behalf of the European Geosciences Union. 
Yokoyama et al. (2004) performed 2-D and 3-D numerical simulations of cloud polarization, current closure, and E-F region coupling, including the recently predicted sporadicE instability (Cosgrove and Tsunoda, 2002b, 2004; Tsunoda et al., 2004; Tsunoda, 2006). These simulations give spatial distributions of the electric field but, being performed for particular cases only, provide no parameter dependencies as we will provide.

In this paper, we study the basic process of the cloud polarization and current closure through the ionosphere, based on a rigorous, albeit simplified, analytic theory. Our objective is to improve the previous theories by developing a selfconsistent analytical approach with reasonable simplifying assumptions. This yields is a reliable parameter dependence of the major characteristics of the process and identifies the most important physical factors. In this paper, we focus on the electrodynamic aspect of cloud polarization, neglecting the feedback of the currents and fields onto the cloud structure and evolution (Rozhansky and Tsendin, 2001; Dimant and Oppenheim, 2006a,b). For simplicity, we assume infinite and homogeneous background ionosphere and external field. In this case, the ionospheric current closure does not include the interaction of the given plasma cloud with other clouds and the F-region ionosphere (Shalimov and Haldoupis, 2005). Also, we do not include wind shears and gravity, that cause large-scale instabilities (Tsunoda and Cosgrove, 2001; Ogawa et al., 2002; Cosgrove and Tsunoda, 2001, 2002a, 2003). As in the majority of other theories, we use a "water-bag" model of the cloud, which implies a uniformly distributed plasma density within a given boundary surface. Instead of a slab model, however, we use a 3-D ellipsoidal ionosphere-cloud interface, which allows a rigorous analytic solution for the electrostatic potential and currents in the entire space. The analytic solution becomes possible via use of renormalized variables and a series of coordinate transformation that reduce the entire plasma problem to a classical solution for an ideally-conducting ellipsoid embedded in vacuum with a given external field. Recently, we have developed such an approach for elongated meteor plasma trails (Dimant et al., 2009). In this paper, we generalize this approach and extend it to pancake-like sporadic-E layers.

The analytic theory of this paper represents a step toward a quantitative understanding of the sporadic-E-ionosphere electrodynamic interaction. The key parameter dependencies identified here can serve for prediction of possible regimes of field and current formation within and around sporadic-E layers. The quantitative estimates of fields obtained in this paper can be used for simple estimates of threshold conditions of instabilities at various spatial scales. This is an important step in understanding coherent radar signals observed at mid- and high latitudes, including midlatitude quasi-periodic echoes. The strong currents predicted to flow through the plasma cloud and the ambient ionosphere can modify the ionospheric conductance at high latitudes, with possible im- plications for global modeling of magnetosphere-ionosphere coupling. The simple analytic theory can serve as a guidance for more accurate and comprehensive studies.

The paper is organized as follows. Section 2 describes our physical model and introduces transformed coordinates for use in the remainder of the paper. Presuming a uniform electric field within the cloud, we give in Sect. 3 a general solution for the electrostatic potential outside it. In Sect. 4, we prove the above presumption, calculate the internal field, and close the entire solution for the general case of oblate ellipsoidal clouds. In Sect. 5, we discuss in detail an important particular case of a round plasma cloud, which can be considered as a reasonable model of a typical sporadic-E layer. Readers interested in a simplified system may skip directly there. Section 6 summarizes our model assumptions and the major results. Appendix A presents the mathematical details of coordinate transformations, while Appendix B discusses an extended (rod-like) cloud, such as, e.g., a meteor plasma trail and finds the plasma density restriction for the elongated-structure solution as presented in Dimant et al. (2009).

\section{Description of general model}

We employ a simplified model of the plasma cloud, approximating it with a uniformly dense pancake-like structure with a sharp boundary. This cloud is embedded in a tenuous homogeneous ionosphere with a uniform DC electric field sustained by an external dynamo. We apply a Cartesian coordinate system with the axes $\boldsymbol{x}_{1}$ and $\boldsymbol{x}_{2}$ in the horizontal plane, while $\boldsymbol{x}_{3}$ is directed along vertical, see Fig. 1. Below, we align the coordinate axes $\boldsymbol{x}_{i}$ with the major axes of the cloud, so that we do not require $\boldsymbol{x}_{1}$ to be directed along the magnetic meridian or some other Earth-related horizontal direction. The geomagnetic field, $\boldsymbol{B}$, is directed at an angle $\theta$ to the horizontal surface (the inclination, or dip, angle), while its horizontal projection has an angle $\varphi$ to the $\boldsymbol{x}_{1}$-axis. While $\theta$ can be arbitrary (without loss of generality, $0^{\circ} \leq \theta \leq 90^{\circ}$ ), we focus on middle and high latitudes, so that $\theta$ is not close to zero. As a result, the external electric field, $\boldsymbol{E}^{\text {ext }} \perp \boldsymbol{B}$, has a significant horizontal component, which plays the major role in the effect under study.

For the interface between the two different plasmas, the dense plasma of the cloud and the tenuous ionospheric plasma, we choose a 3-D ellipsoid with the principal axes aligned with the coordinate axes,

$$
\frac{x_{1}^{2}}{a_{1}^{2}}+\frac{x_{2}^{2}}{a_{2}^{2}}+\frac{x_{3}^{2}}{a_{3}^{2}}=1 .
$$

For sporadic-E layers, we assume that the vertical thickness of the ellipsoid is much less than the two horizontal semiaxes,

$a_{3} \ll a_{2} \leq a_{1}$. 


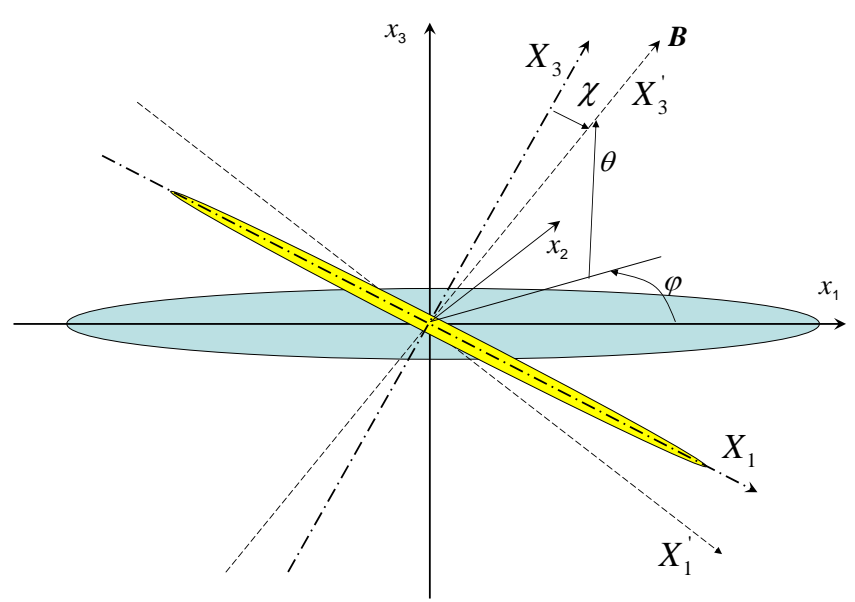
original cloud is shaded blue. Axes $x_{1,2,3}$ are aligned with the principal semi-axes of ellipsoid; axis $X_{3}^{\prime}$ is along $\boldsymbol{B}, X_{2}^{\prime}$ (not shown) is perpendicular to $x_{3}$ and $X_{3}^{\prime}$, and axis $X_{1}^{\prime}$ is perpendicular to $X_{2}^{\prime}$ and $X_{3}^{\prime}$. Further, $\theta$ is the angle between $x_{1}$ and $\boldsymbol{B}$, while $\varphi$ is the angle between the $x_{3} X_{3}^{\prime}$-plane. The cloud rescaled by $\epsilon$, Eq. (7), is shaded yellow; $\chi$ is the angle between $X_{3}^{\prime}$ and $X_{3}$; the final axes $X_{k}$ are aligned with the principal axes of the rescaled ellipsoid.

In Dimant et al. (2009) we assumed that for meteor plasma trails $a_{3} \sim a_{2} \ll a_{1}$. As shown in Sect. B, the more general results obtained in this paper cover that case as well. Unlike meteor trails which are symmetric with respect to the magnetic field in the trail cross-section (Dimant and Oppenheim, 2006a), the alignment of the sporadic-E layer ellipsoid with respect to $\boldsymbol{B}$ is arbitrary (without loss of generality, $0 \leq \varphi \leq 90^{\circ}$ ).

In this paper, we calculate the distributions of the electrostatic fields, $\boldsymbol{E}=-\nabla \Phi$, and current densities, $\boldsymbol{j}=\overleftrightarrow{\sigma} \boldsymbol{E}$, within and around a given plasma cloud, rather than the feedback of the developed fields to the cloud structure and evolution (Rozhansky and Tsendin, 2001; Dimant and Oppenheim, 2006a,b). Here, $\Phi$ is the electrostatic potential and $\overleftrightarrow{\sigma}$ is the conductivity tensor proportional to the plasma density. The spatial scales of these distributions are usually many orders of magnitude larger that the typical Debye lengths, so that our electrostatic problem reduces to solving the quasineutrality equation,

$\nabla \cdot \boldsymbol{j} \equiv-\nabla \cdot(\overleftrightarrow{\sigma} \nabla \Phi)=0$

in a conducting medium with an imposed external electric field, $\boldsymbol{E}^{\mathrm{ext}}$, sustained by an external dynamo. $115 \mathrm{~km}$ of an altitude, electrons are strongly magnetized, $v_{e} \ll \Omega_{e}$, while ions are unmagnetized, $v_{i} \gg \Omega_{i}$. Here, $v_{e, i}$ are the mean rates of electron-neutral and ion-neutral collisions, and $\Omega_{e, i}=e B / m_{e, i}$ are the electron and single-charge ion gyrofrequencies, where $e$ is the elementary charge, $m_{e, i}$
Fig. 1. Schematic geometry of the cloud and successive coordinate transformations $x_{i} \rightarrow X_{k}$ (described in detail in Appendix A). The

In the upper D/lower E region ionosphere between 80 and

are the electron and average ion masses, $m_{i} \simeq 30 \mathrm{amu}$, and, for this study, we use $v_{e} \simeq 10 v_{i}$. Under these conditions, the electron response to the electric field is sharply anisotropic with respect to the direction of the magnetic field. In a plasma with a given density $n \equiv n_{e} \approx n_{i}$, the total current densities are given by

$\boldsymbol{j}_{\|}=\sigma_{\|} \boldsymbol{E}_{\|}$,

$\boldsymbol{j}_{\perp}=\sigma_{\mathrm{P}} \boldsymbol{E}_{\perp}+\sigma_{\mathrm{H}} \boldsymbol{E}_{\perp} \times \hat{b}$,

where $\boldsymbol{E}_{\|}=-\nabla_{\|} \Phi, \boldsymbol{E}_{\perp}=-\nabla_{\perp} \Phi$, and $\hat{b}$ is the unit vector along $\boldsymbol{B}$. In the fluid approximation, the total parallel, Pedersen, and Hall conductivities at the altitudes of interest are given by

$$
\begin{aligned}
\sigma_{\|} & \approx \frac{n e^{2}}{m_{e} v_{e}}=\frac{n e}{B \Theta_{0} \sqrt{\psi}}, \\
\sigma_{\mathrm{P}} & \approx \frac{n e^{2}}{m_{i} v_{i}}+\frac{n e^{2} v_{e}}{m_{e} \Omega_{e}^{2}}=\frac{(1+\psi) \Theta_{0} n e}{B \sqrt{\psi}}, \\
\sigma_{\mathrm{H}} & \approx \frac{n e}{B},
\end{aligned}
$$

where (e.g., Dimant and Oppenheim, 2006a)

$$
\psi=\frac{v_{e} v_{i}}{\Omega_{e} \Omega_{i}}, \quad \Theta_{0}=\left(\frac{m_{e} v_{e}}{m_{i} v_{i}}\right)^{1 / 2} \simeq 1.35 \times 10^{-2} .
$$

To solve Eq. (3), we will perform a series of coordinate transformations similar to those made by Dimant et al. (2009). These transformations consist of several sequential steps described in detail in Appendix A. First, we rotate the coordinate system in order to align one of the new coordinate axes with the magnetic field. Second, we rescale the corresponding coordinate to make the total parallel and Pedersen conductivities into Eq. (3) equal. This results in an effective rescaling and rotation of the ellipsoidal cloud, so that the ellipsoid equation loses its simple canonical form. To restore it, we make a final coordinate rotation, which aligns the coordinate axes with the principal axes of the rescaled ellipsoid. To the first-order accuracy with respect to small parameters

$\epsilon \equiv\left(\frac{\sigma_{\mathrm{P}}}{\sigma_{\|}}\right)^{1 / 2} \approx \Theta_{0}(1+\psi)^{1 / 2} \ll 1$

and $a_{3} / a_{1,2}$, see Eq. (2), the entire coordinate transformations from $x_{i}$ to the final coordinates $X_{k}, X_{k}=$ $\sum_{i=1}^{3}\left(\partial X_{k} / \partial x_{i}\right) x_{i}$, and back, $x_{i}=\sum_{k=1}^{3}\left(\partial x_{i} / \partial X_{k}\right) X_{k}$, are given in matrix form by

$$
\begin{aligned}
& {\left[\frac{\partial X_{k}}{\partial x_{i}}\right] \equiv\left[\begin{array}{lll}
\partial X_{1} / \partial x_{1} & \partial X_{1} / \partial x_{2} & \partial X_{1} / \partial x_{3} \\
\partial X_{2} / \partial x_{1} & \partial X_{2} / \partial x_{2} & \partial X_{2} / \partial x_{3} \\
\partial X_{3} / \partial x_{1} & \partial X_{3} / \partial x_{2} & \partial X_{3} / \partial x_{3}
\end{array}\right]} \\
& \approx\left[\begin{array}{ccc}
\sin \eta \sin \varphi+\cos \eta \sin \theta \cos \varphi & \cos \eta \sin \varphi \sin \theta-\sin \eta \cos \varphi & -\cos \eta \cos \theta \\
\sin \eta \sin \theta \cos \varphi-\cos \eta \sin \varphi & \cos \eta \cos \varphi+\sin \eta \sin \theta \sin \varphi & -\sin \eta \cos \theta \\
0 & 0 & \epsilon / \sin \theta
\end{array}\right]
\end{aligned}
$$


and

$\left[\frac{\partial x_{i}}{\partial X_{k}}\right] \equiv\left[\begin{array}{lll}\partial x_{1} / \partial X_{1} & \partial x_{1} / \partial X_{2} & \partial x_{1} / \partial X_{3} \\ \partial x_{2} / \partial X_{1} & \partial x_{2} / \partial X_{2} & \partial x_{2} / \partial X_{3} \\ \partial x_{3} / \partial X_{1} & \partial x_{3} / \partial X_{2} & \partial x_{3} / \partial X_{3}\end{array}\right]$

$\approx\left[\begin{array}{ccc}\frac{\cos \eta \cos \varphi+\sin \eta \sin \theta \sin \varphi}{\sin \theta} & \frac{\sin \eta \cos \varphi-\cos \eta \sin \theta \sin \varphi}{\sin \theta} & \frac{\cos \theta \cos \varphi}{\epsilon} \\ \frac{\cos \eta \sin \varphi-\sin \eta \sin \theta \cos \varphi}{\sin \theta} & \frac{\sin \eta \sin \varphi+\cos \eta \sin \theta \cos \varphi}{\sin \theta} & \frac{\cos \theta \sin \varphi}{\epsilon} \\ 0 & 0 & \frac{\sin \theta}{\epsilon}\end{array}\right]$

Here the angle $\eta$, in the range of $0 \leq \eta \leq 180^{\circ}$, is uniquely determined by

$\tan 2 \eta=\frac{2 \tan \eta}{1-\tan ^{2} \eta} \approx \frac{2\left(1-a_{2}^{2} / a_{1}^{2}\right) \sin \theta \tan \varphi}{\sin ^{2} \theta-\tan ^{2} \varphi-a_{2}^{2}\left(1-\sin ^{2} \theta \tan ^{2} \varphi\right) / a_{1}^{2}}$.

The Jacobians of these transformations are given by

$\operatorname{det}\left[\frac{\partial X_{k}}{\partial x_{i}}\right]=\left(\operatorname{det}\left[\frac{\partial x_{i}}{\partial X_{k}}\right]\right)^{-1}=\epsilon$

so that the renormalized plasma density in the transformed coordinates, $N$, is related to the original plasma density, $n$, as $N=n / \epsilon$. The current density transforms according to $J_{X_{k}}=\operatorname{det}\left[\partial x_{i} / \partial X_{k}\right] \times \sum_{i=1}^{3}\left(\partial X_{k} / \partial x_{i}\right) J_{i}=$ $(1 / \epsilon) \sum_{i=1}^{3}\left(\partial X_{k} / \partial x_{i}\right) J_{i}$, while the electric field transforms according to $E_{X_{k}}=\sum_{i=1}^{3}\left(\partial x_{i} / \partial X_{k}\right) E_{i}$, where $E_{X_{k}}$ and $J_{X_{k}}$ are the $X_{k}$-components of the electric field and current density, respectively, and $E_{i}$ and $J_{i}$ are the corresponding components in real coordinates, $x_{i}$. We note that $\boldsymbol{E}_{\perp}=\nabla_{\perp} \Phi$ responds only to the coordinate rotations and remain unaffected by the coordinate rescaling along $\boldsymbol{B}$. In particular, the total magnitude of the external electric field, $\boldsymbol{E}^{(0)} \perp \boldsymbol{B}$, remains invariant with respect to the entire coordinate transformation from $x_{i}$ to $X_{k},\left|\boldsymbol{E}^{(0)}\right|=\left|\boldsymbol{E}_{\boldsymbol{X}}^{(0)}\right|$. At the same time, $E_{\|}=-\nabla_{\|} \Phi$ increases in the new $X_{k}$-coordinates by a large factor of $\epsilon^{-1}$.

In the $X_{k}$-coordinates, the cloud boundary remains an ellipsoid with the rescaled semi-axes,

$\frac{X_{1}^{2}}{A_{1}^{2}}+\frac{X_{2}^{2}}{A_{2}^{2}}+\frac{X_{3}^{2}}{A_{3}^{2}}=1$

where

$$
\begin{aligned}
& A_{1} \approx \frac{\sin \theta}{\cos \varphi \cos \eta}\left[\frac{(1+\sin \theta \tan \varphi \tan \eta)^{2}}{a_{1}^{2}}+\frac{(\tan \varphi-\sin \theta \tan \eta)^{2}}{a_{2}^{2}}\right]^{-1 / 2}(13 \mathrm{a}) \\
& A_{2} \approx \frac{\sin \theta}{\cos \varphi \cos \eta}\left[\frac{(\tan \eta-\sin \theta \tan \varphi)^{2}}{a_{1}^{2}}+\frac{(\sin \theta+\tan \varphi \tan \eta)^{2}}{a_{2}^{2}}\right]^{-1 / 2} \\
& A_{3} \approx \frac{\epsilon a_{3}}{\sin \theta} .
\end{aligned}
$$

The restrictions of Eqs. (2) and (7) make the rescaled ellipsoid even more flattened than it was in the original coordinates,

$A_{3} \lll A_{2} \lesssim A_{1}$.

One can check that Eqs. (10) and (13) simplify drastically in certain limiting cases. For $\theta \approx 90^{\circ}$, we have $\eta \approx \varphi, A_{1} \approx a_{1}$, and $A_{2} \approx a_{2}$. For a disk-like ellipsoid, $a_{1}=a_{2}$ (or for an arbitrary ellipsoid, but $\varphi=0$ ), we have $\eta=0, A_{1} \approx a_{1} \sin \theta$, and $A_{2} \approx a_{2}$. In the opposite limit of an extended cloud, $a_{1} \gg a_{2}$, we obtain $\tan \eta \approx \tan \varphi / \sin \theta, A_{1} \approx a_{1}\left(1-\cos ^{2} \theta \cos ^{2} \varphi\right)^{1 / 2}$, and $A_{2} \approx a_{2}\left(1-\cos ^{2} \theta \cos ^{2} \varphi\right)^{-1 / 2} \sin \theta$.

In the new coordinates, the renormalized current density, $J_{\boldsymbol{X}}$, is described in Appendix A by Eqs. (A11) to (A13), so that Eq. (3) becomes in renormalized form

$\nabla_{X} \cdot J_{X}=0$,

where $\nabla_{X}$ is the nabla operator in $X_{i}$, and reduces to Laplace's equation for the potential,

$\nabla_{X}^{2} \Phi=0$.

In Sect. 3, we solve Eq. (16) outside the dense plasma cloud, i.e., in the background ionosphere.

Far from the cloud, the electrostatic electric field, $\boldsymbol{E}=$ $-\nabla \Phi$, approaches asymptotically the external field, $\boldsymbol{E}^{\text {ext }}$. Within the cloud, we initially presume a uniform electric field, $\boldsymbol{E}^{\text {int }}$, so that on the ellipsoidal interface which separates the dense cloud from the tenuous ionosphere $\Phi$ approaches a linearly dependent potential. The spaces inside and outside the cloud are related through conservation of currents that are collected from the ionosphere into one semi-cloud, flow through the cloud, and release back to the ionosphere from the other semi-cloud, see Fig. 2. In Sect. 4, we calculate the fields and currents within the cloud and show that our presumption of the uniform electric field (and, hence, of the uniform current density) within the cloud is consistent with the ellipsoidal interface and the homogeneous plasma density. This allows us to calculate the internal electric field and thus close the entire solution.

\section{External fields and currents}

In this section, we solve Eq. (16) outside the ellipsoid, assuming a given potential $\Phi=\Phi^{\text {int }} \equiv-\boldsymbol{E}^{\text {int }} \cdot \boldsymbol{X}$ on the cloudionosphere interface and the asymptotic boundary condition, $\Phi \rightarrow-\boldsymbol{E}^{\mathrm{ext}} \cdot \boldsymbol{X}$ far from the interface. Similarly to Dimant et al. (2009), we extrapolate the internal linear potential to the entire space and introduce $\Delta \Phi \equiv \Phi-\Phi^{\text {int }}$. On the ellipsoidal interface, $\Delta \Phi=0$, while far from the cloud $\Delta \Phi$ asymptotically approaches $\left(\boldsymbol{E}^{\mathrm{int}}-\boldsymbol{E}^{\mathrm{ext}}\right) \cdot \boldsymbol{X}$. This reduces the original problem of finding the potential in a highly anisotropic, weakly conducting plasmas to a classic problem of finding 
the potential around an infinitely conducting ellipsoid embedded in vacuum with a given uniform external field (e.g., Landau and Lifshitz, 1960). Dimant et al. (2009) describes this approach in detail, so here we only present the crucial steps and give the results.

The solution for $\Delta \Phi=0$ is given by a linear superposition of partial potentials,

$$
\Delta \Phi=\sum_{i=1}^{3} \Delta \Phi^{(i)}
$$

where each partial potential is a solution of Laplace's equation with the boundary conditions corresponding to one component of $\left(E_{X_{i}}^{\mathrm{int}}-E_{X_{i}}^{(0)}\right)$ far from the cloud. Hence, $\Delta \Phi^{(i)} \rightarrow$ $\left(E_{X_{i}}^{\mathrm{int}}-E_{X_{i}}^{(0)}\right) X_{i}$ as $\left|X_{i}\right| \rightarrow \infty$, and $\Delta \Phi^{(i)}=0$ on the ellipsoid surface defined by Eq. (12). Following the procedure outlined in Landau and Lifshitz (1960); Dimant et al. (2009), Laplace's equation for $\Delta \Phi^{(i)}$ is solved in terms of ellipsoidal coordinates defined as the three independent real roots of the cubic equation

$$
\frac{X_{1}^{2}}{A_{1}^{2}+u}+\frac{X_{2}^{2}}{A_{2}^{2}+u}+\frac{X_{3}^{2}}{A_{3}^{2}+u}=1 .
$$

The largest (and the only positive) root, $u=\xi$, becomes zero on the boundary surface described by Eq. (12) and tends to infinity as $\left|X_{i}\right| \rightarrow \infty$. The exact solution for a partial potential is given by

$$
\Delta \Phi^{(i)}=\frac{\left(E_{X_{i}}^{\mathrm{int}}-E_{X_{i}}^{(0)}\right) X_{i} A_{i}^{3}}{2 \Lambda_{i}} \int_{0}^{\xi} \frac{d \xi}{\left(A_{i}^{2}+\xi\right) R_{\xi}}
$$

where

$$
\begin{aligned}
& R_{\xi}=\left[\left(\xi+A_{1}^{2}\right)\left(\xi+A_{2}^{2}\right)\left(\xi+A_{3}^{2}\right)\right]^{1 / 2}, \\
& \Lambda_{i}=\frac{A_{i}^{3}}{2} \int_{0}^{\infty} \frac{d \xi}{\left(A_{i}^{2}+\xi\right) R_{\xi}} .
\end{aligned}
$$

The integrals in Eqs. (19) and (20b) can be expressed in terms of canonical elliptic integrals, as described in Appendix B of Dimant et al. (2009).

Before going any further, we note that results of our specific model should obey one important general relation. At distances from the cloud much larger than the cloud size, the electrostatic potential of the totally neutral, but polarized, cloud should have asymptotically a dipole character. In the renormalized coordinates $\boldsymbol{X}$, the entire structure of fields and currents is determined by the solution of Laplace's Eq. (16). Then in terms of the dipole moment, $\boldsymbol{D}$, induced in the cloud by the external electric field, $\boldsymbol{E}_{\boldsymbol{X}}^{(0)}$, the total asymptotic potential is be given by

$\Phi(\boldsymbol{X}) \approx-\boldsymbol{E}_{\boldsymbol{X}}^{(0)} \cdot \boldsymbol{X}+\frac{\boldsymbol{D} \cdot \boldsymbol{X}}{X^{3}}$
It is important that this is a universal expression, in which the specific geometry and internal structure of the distant cloud are hidden within $\boldsymbol{D}$. As one might expect, our specific solution for the waterbag ellipsoidal cloud also reduces to Eq. (21). Indeed, in the far zone defined by $X \equiv$ $\left(\sum_{i=1}^{3} X_{i}^{2}\right)^{1 / 2} \gg A_{1} \geq A_{2,3}$, we have $R \approx \xi^{3 / 2}$ and $\xi \approx X^{2}$, so that Eq. (19) can be written asymptotically as

$\Delta \Phi^{(i)} \approx\left(E_{X_{i}}^{\mathrm{int}}-E_{X_{i}}^{(0)}\right)\left(1-\frac{A_{i}^{3}}{3 \Lambda_{i} X^{3}}\right) X_{i}$.

Following Landau and Lifshitz (1960), we introduce the individual components of $\boldsymbol{D}$ as

$D_{i} \equiv \frac{\left(E_{X_{i}}^{(0)}-E_{X_{i}}^{\mathrm{int}}\right) A_{i}^{3}}{3 \Lambda_{i}}=\frac{\left(E_{X_{i}}^{(0)}-E_{X_{i}}^{\mathrm{int}}\right) V}{4 \pi n_{i}}$,

where $V=4 \pi A_{1} A_{2} A_{3} / 3$ is the ellipsoid volume (in the $X$ coordinates) and $n_{i}$ are standard depolarization coefficients closely related to our coefficients $\Lambda_{i}$. Then the total electrostatic potential, $\Phi \equiv \sum_{i=1}^{3}\left(-E_{X_{i}}^{\mathrm{int}} X_{i}+\Delta \Phi^{(i)}\right)$, satisfies Eq. (21).

In addition to the continuity of the potential through the ionosphere-cloud interface, the plasma cloud interacts with the ambient ionosphere through the inflowing and outflowing currents. Given the electric field, the current density is determined by Eqs. (A11) and (A12) in Appendix A. The components of the total field are $E_{X_{j}}^{\text {int }}+\Delta E_{X_{j}}$, where $\Delta E_{X_{j}}=-\partial \Delta \Phi / \partial X_{j}$ is the induced electric field. Note that our definition of the "induced" field differs from the definition of the "polarization" field by Shalimov et al. (1998), which in our notation would be $E_{X_{j}}-E_{X_{j}}^{(0)}$. The predominantly Hall response to the internal field $E_{X_{j}}^{\text {int }}$ on the cloud boundary is unimportant for the total current balance because the corresponding currents on the opposite symmetric sides of the ellipsoid cancel each other (these currents may slightly distort the cloud shape and affect cloud's overall drift, but these effects are beyond the scope of the present paper). The induced electric field $\Delta E_{X_{j}}$ on the ionosphere side of the ellipsoidal interface plays the crucial role in the ionospherecloud current transfer. Since there $\Delta \Phi=0$ this field is normal to the interface. The unit vector normal to the ellipsoidal interface equals $X_{j} /\left(A_{j}^{2} \sqrt{P}\right)$, where

$P \equiv \sum_{i=1}^{3} \frac{X_{i}^{2}}{A_{i}^{4}} \approx \frac{X_{3}^{2}}{A_{3}^{4}}$

On the interface we have

$\Delta E_{X_{j}}=\frac{X_{j}}{A_{j}^{2} \sqrt{P}} \sum_{i=1}^{3} \Delta E^{(i)}$, 
where $\Delta E^{(i)}$ are the partial components of the induced-field amplitude,

$$
\begin{aligned}
\Delta E^{(i)} & =-\left.\frac{2}{\sqrt{P}} \frac{\partial \Delta \Phi^{(i)}}{\partial \xi}\right|_{\xi=0}=-\frac{\left(E_{X_{i}}^{\mathrm{int}}-E_{X_{i}}^{(0)}\right) X_{i} A_{i}}{\Lambda_{i} A_{1} A_{2} A_{3} \sqrt{P}} \\
& \approx-\frac{\left(E_{X_{i}}^{\mathrm{int}}-E_{X_{i}}^{(0)}\right) X_{i} A_{i} A_{3}}{\Lambda_{i} A_{1} A_{2} X_{3}} .
\end{aligned}
$$

The approximate equality in the right-hand side (RHS) of Eq. (24) is valid on most of the ellipsoidal surface, excluding a narrow vicinity of the largest central cross-section $X_{1}^{2} / A_{1}^{2}+$ $X_{2}^{2} / A_{2}^{2}=0$ where $X_{3} / A_{3} \lesssim A_{3} / A_{1,2} \lll 1$. On most of the surface we have $\Delta E_{X_{1,2}} \approx X_{1,2} A_{3}^{2} \Delta E_{X_{3}} /\left(X_{3} A_{1,2}^{2}\right) \ll$ $\Delta E_{X_{3}} \approx \sum_{i=1}^{3} \Delta E^{(i)}$, so that the total induced electric field there is directed practically parallel to $\boldsymbol{X}_{3}$ with the magnitude given by

$$
\begin{aligned}
\left|\Delta \boldsymbol{E}_{\boldsymbol{X}_{i}}\right| & \approx \Delta E_{X_{3}} \\
& \approx-\frac{A_{3}}{X_{3}}\left[\frac{\left(E_{X_{1}}^{\mathrm{int}}-E_{X_{1}}^{(0)}\right) X_{1}}{\Lambda_{1} A_{2}}+\frac{\left(E_{X_{2}}^{\mathrm{int}}-E_{X_{2}}^{(0)}\right) X_{2}}{\Lambda_{2} A_{1}}\right]
\end{aligned}
$$

In Eq. (26) we neglected a contribution to the induced field that comes from a relatively small field component $\left(E_{X_{3}}^{\mathrm{int}}-\right.$ $\left.E_{X_{3}}^{(0)}\right)$. On the ionosphere-cloud interface corresponding to

$X_{3}=X_{3 B}\left(X_{1,2}\right) \equiv A_{3}\left(1-\frac{X_{1}^{2}}{A_{1}^{2}}-\frac{X_{2}^{2}}{A_{2}^{2}}\right)^{1 / 2}$,

the normal component of the ionospheric current density, $J_{n}^{\text {ext }} \mid X_{3 B}$, continues through the interface. On most of the surface, this component is directed mainly along $\boldsymbol{X}_{3}$ and is determined by the $X_{3}$-component of the field. Equations (A11) and (A12) from Appendix A give $J_{X_{3}}^{\mathrm{ext}}=K^{\mathrm{ext}} \Delta E_{X_{3}}$, so that

$\left.J_{n}^{\mathrm{ext}}\right|_{X_{3 B}} \approx A_{3} K^{\mathrm{ext}}\left[\frac{\left(E_{X_{1}}^{(0)}-E_{X_{1}}^{\mathrm{int}}\right) X_{1}}{\Lambda_{1} A_{2} X_{3 B}}+\frac{\left(E_{X_{2}}^{(0)}-E_{X_{2}}^{\mathrm{int}}\right) X_{2}}{\Lambda_{2} A_{1} X_{3 B}}\right]$,

where, in accord with Eq. (A6a),

$K^{\mathrm{ext}}=\left(\frac{1+\psi}{\psi}\right)^{1 / 2} \frac{e n_{0}}{B}$

(recall that $n_{0}$ is the ionospheric density measured in the original coordinate system, $x_{i}$ ). In the next section, we relate the external ionospheric currents to the internal currents on the cloud side of the ionosphere-cloud interface.

\section{Internal fields and currents; closure of entire solution}

In the previous analysis, we presumed a uniform electric field and current density inside the ellipsoidal plasma cloud. As-

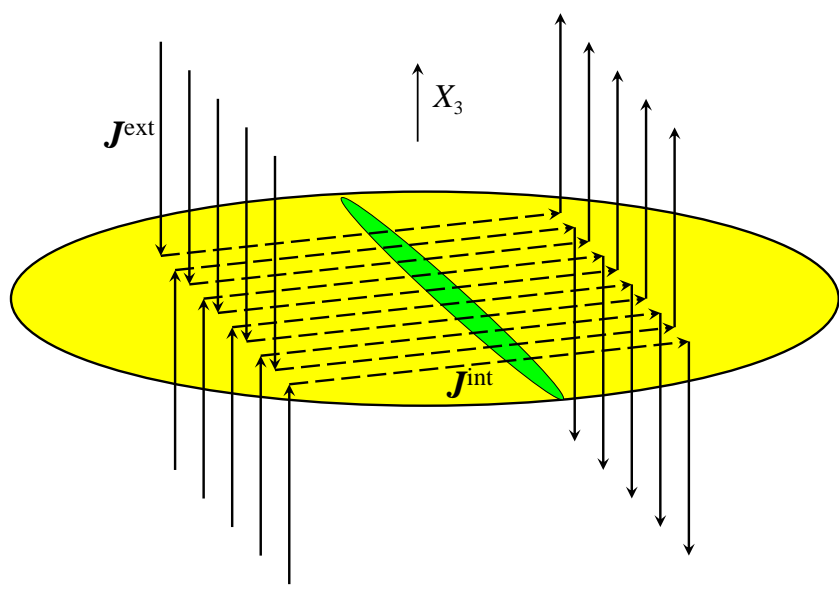

Fig. 2. Cartoon of ionospheric current closure through an oblate cloud (shaded yellow). The vertical solid lines $\left(\boldsymbol{J}^{\text {ext }}\right)$ depict the ionospheric (external) current flow, while the horizontal dashed lines $\left(\boldsymbol{J}^{\text {int }}\right)$ depict the internal current flow through the cloud. Under the assumed conditions, the current flow has an up-down symmetry with respect to the central horizontal plane, $X_{3}=0$. The current flow is antisymmetric with respect to the central cross-section of the cloud by the vertical plane normal to the internal current flow (shaded green).

suming also a mirror symmetry of the current flow with respect to the central plane $X_{3}=0$, we see that the internal currents within the highly conducting pancake-like cloud should flow only horizontally, $J_{X_{3}}^{\text {int }}=0$, as illustrated in Fig. 2 . On the boundary surface, the normal component of the internal current, $\left.J_{n}^{\text {int }}\right|_{X_{3 B}}$, equals the corresponding component of the external current, $\left.\left.J_{n}^{\mathrm{ext}}\right|_{X_{3 B}} \approx J_{X_{3}}^{\text {ext }}\right|_{X_{3 B}}$. Unlike the external currents that flow mainly along $\boldsymbol{X}_{3}$, the normal component of the internal current is relatively small on most of the boundary surface where $X_{3 B} / A_{3} \gg A_{3} / A_{1,2}$,

$\left.J_{n}^{\mathrm{int}}\right|_{X_{3 B}}=\left.\hat{n} \cdot \boldsymbol{J}^{\mathrm{int}}\right|_{X_{3 B}} \approx \frac{A_{3}^{2}}{X_{3 B}}\left(\frac{X_{1} J_{X_{1}}^{\mathrm{int}}}{A_{1}^{2}}+\frac{X_{2} J_{X_{2}}^{\mathrm{int}}}{A_{2}^{2}}\right)$,

where $X_{3 B}\left(X_{1,2}\right)$ is defined by Eq. (27). The internal current components $J_{X_{1,2}}^{\text {int }}$, in terms of the internal electric field, are given by Eqs. (A11) and (A12) with

$K=K^{\mathrm{int}} \equiv\left(\frac{1+\psi}{\psi}\right)^{1 / 2} \frac{e n^{\mathrm{int}}}{B}$

where $n^{\text {int }} \gg n_{0}$ is the uniform cloud plasma density. Setting $J_{X_{3}}^{\text {int }}=0$, we obtain

$E_{X_{3}}^{\mathrm{int}}=-p\left(E_{X_{1}}^{\mathrm{int}} \sin \eta-E_{X_{2}}^{\mathrm{int}} \cos \eta\right)$,

where $p$ is defined by Eq. (A13) with $q$ defined by Eq. (A6b). Note that Shalimov et al. (1998) and Shalimov and Haldoupis (2005) disregarded the corresponding vertical component of 
the polarization electric field inside the layer (it would be $E_{z}^{p}$ in their notations), implying no slab polarization in the vertical direction. However, in the general case of $\theta \neq 90^{\circ}$ (i.e., $p \neq 0)$, neglecting this component will lead to substantial errors. The resultant currents from Eq. (32) become

$$
\begin{aligned}
& J_{X_{1}}^{\text {int }}=\frac{K^{\mathrm{ext}} A_{1}^{2}\left(\alpha_{1} E_{X_{1}}^{\mathrm{int}}+\gamma_{1} E_{X_{2}}^{\mathrm{int}}\right)}{\Lambda_{1} A_{2} A_{3}}, \\
& J_{X_{2}}^{\mathrm{int}}=\frac{K^{\mathrm{ext}} A_{2}^{2}\left(\alpha_{2} E_{X_{2}}^{\mathrm{int}}-\gamma_{2} E_{X_{1}}^{\mathrm{int}}\right)}{\Lambda_{2} A_{1} A_{3}},
\end{aligned}
$$

where we used, according to Eqs. (29) and (31), $K^{\text {int }} / K^{\text {ext }}=$ $n^{\text {int }} / n_{0}$ and introduced

$\begin{aligned} \alpha_{1} & =\frac{\Lambda_{1} A_{2} A_{3}\left(1+p^{2} \sin ^{2} \eta\right) n^{\mathrm{int}}}{A_{1}^{2} n_{0}}, \\ \alpha_{2} & =\frac{\Lambda_{2} A_{1} A_{3}\left(1+p^{2} \cos ^{2} \eta\right) n^{\mathrm{int}}}{A_{2}^{2} n_{0}},\end{aligned}$

$\gamma_{1}=\frac{\Lambda_{1} A_{2} A_{3}\left(q \cos \chi-p^{2} \sin \eta \cos \eta\right) n^{\text {int }}}{A_{1}^{2} n_{0}}$,

$\gamma_{2}=\frac{\Lambda_{2} A_{1} A_{3}\left(q \cos \chi+p^{2} \sin \eta \cos \eta\right) n^{\text {int }}}{A_{2}^{2} n_{0}}$.

Here $q$ and $p$ are defined by Eqs. (A6b) and (A13). As a result, we obtain from Eqs. (30) and (33)

$$
\left.J_{n}^{\mathrm{int}}\right|_{X_{3 B}} \approx A_{3} K^{\mathrm{ext}}\left[\frac{\left(\alpha_{1} E_{X_{1}}^{\mathrm{int}}+\gamma_{1} E_{X_{2}}^{\mathrm{int}}\right) X_{1}}{\Lambda_{1} A_{2} X_{3 B}}+\frac{\left(\alpha_{2} E_{X_{2}}^{\mathrm{int}}-\gamma_{2} E_{X_{1}}^{\mathrm{int}}\right) X_{2}}{\Lambda_{2} A_{1} X_{3 B}}\right] .
$$

Now, we equate $\left.J_{n}^{\text {int }}\right|_{X_{3 B}}$ to $\left.J_{n}^{\text {ext }}\right|_{X_{3 B}}$ from Eq. (28). The RHSs of Eqs. (28) and (36) have similar structures of their coordinate-dependent terms proportional to $X_{1,2} / X_{3 B}$. This ensures that the uniform internal field is really the proper solution for a uniformly dense ellipsoidal cloud. The spatial dependence of the terms allows one to properly split the equation $\left.J_{n}^{\text {int }}\right|_{X_{3 B}}=\left.J_{n}^{\text {ext }}\right|_{X_{3 B}}$ and calculate separate internal field components.

Note that Eq. (3) of Shalimov et al. (1998) is split into their Eqs. (4) and (5) without justification and we argue that this may lead to highly inaccurate estimate for the fields. Their splitting is based on the idea that two independent currents flow in the east-west and north-south directions due to the potential differences caused by charge accumulation at opposite boundaries of the assumed slab layer. This argument never works, regardless of the specific cloud geometry. The reason is that the current system outside the cloud satisfies $\nabla \cdot \boldsymbol{J}=0$ which reduces to a single second-order differential equation for the electrostatic potential, like Eq. (16). If one considers a uniform ionosphere at distances much larger than the maximum cloud size then the corresponding asymptotic solution is always expressed in terms of the induced dipole moment of the cloud, $\boldsymbol{D}$, as described by Eq. (21). Given $\boldsymbol{D}$, the total electric field is determined by the well-known expression $\boldsymbol{E}_{\boldsymbol{X}} \approx \boldsymbol{E}_{\boldsymbol{X}}^{(0)}+\left[3 \boldsymbol{X}(\boldsymbol{D} \cdot \boldsymbol{X})-X^{2} \boldsymbol{D}\right] / X^{5}$, so that the far-zone ionospheric current system is fully described by Eq. (A11). Expressed in terms of $\boldsymbol{D}$, the dipolar current flow system is insensitive to the details of the geometry and internal structure of the distant cloud. These assumptions also apply to the slab geometry considered by Shalimov et al. (1998). The general asymptotic solution results in a structure of currents that can never be split into two independent systems, like the zonal and meridional current flows. The currents in the asymptotic remote regions represent only a part of the entire ionospheric current-closure system, but there is no reason to expect a fundamentally different behavior in the near-cloud zone. Moreover, our exact solution for the ellipsoidal cloud in terms of the coordinate $\xi$ given by Eq. (19) clearly shows that there are not two independent current systems.

We will now proceed with calculating the fields. Equating in the RHSs of Eqs. (28) and (36) the corresponding coefficients in front of $X_{1,2} / X_{3 B}$, we obtain a set of two linear equations,

$$
\begin{array}{r}
\left(1+\alpha_{1}\right) E_{X_{1}}^{\text {int }}+\gamma_{1} E_{X_{2}}^{\text {int }} \approx E_{X_{1}}^{(0)}, \\
-\gamma_{2} E_{X_{1}}^{\text {int }}+\left(1+\alpha_{2}\right) E_{X_{2}}^{\text {int }} \approx E_{X_{2}}^{(0)},
\end{array}
$$

with the solution given by

$$
\begin{aligned}
& E_{X_{1}}^{\mathrm{int}} \approx \frac{\left(1+\alpha_{2}\right) E_{X_{1}}^{(0)}-\gamma_{1} E_{X_{2}}^{(0)}}{\left(1+\alpha_{1}\right)\left(1+\alpha_{2}\right)+\gamma_{1} \gamma_{2}}, \\
& E_{X_{2}}^{\mathrm{int}} \approx \frac{\left(1+\alpha_{1}\right) E_{X_{2}}^{(0)}+\gamma_{2} E_{X_{1}}^{(0)}}{\left(1+\alpha_{1}\right)\left(1+\alpha_{2}\right)+\gamma_{1} \gamma_{2}} .
\end{aligned}
$$

For small $\alpha_{i}$ and $\gamma_{i}$, the internal electric field, $\boldsymbol{E}_{\boldsymbol{X}}^{\text {int }}$, approaches the external field, $\boldsymbol{E}_{\boldsymbol{X}}^{(0)}$. In the general case, the direction of the internal field, $\boldsymbol{E}_{\boldsymbol{X}}^{\text {int }}$, is rotated with respect to that of the external field, $\boldsymbol{E}_{X}^{(0)}$, through an angle $\phi_{E}$ determined by

$$
\left|\tan \phi_{E}\right| \approx \frac{\gamma_{2}\left(E_{X_{1}}^{(0)}\right)^{2}+\left(\alpha_{1}-\alpha_{2}\right) E_{X_{1}}^{(0)} E_{X_{2}}^{(0)}+\gamma_{1}\left(E_{X_{2}}^{(0)}\right)^{2}}{\left(1+\alpha_{2}\right)\left(E_{X_{1}}^{(0)}\right)^{2}+\left(\gamma_{2}-\gamma_{1}\right) E_{X_{1}}^{(0)} E_{X_{2}}^{(0)}+\left(1+\alpha_{1}\right)\left(E_{X_{2}}^{(0)}\right)^{2}} .
$$

The sign of $\phi_{E}$ is determined by the direction of $\boldsymbol{B}$. In the Northern Hemisphere, where the magnetic field is directed downward, the internal field is rotated with respect to the external field anti-clockwise when looking from above.

Combining Eqs. (38) and Eq. (32) gives the previously unknown components of the internal electric field and thus closes the solution for the potential and currents in the entire space. Using

$$
\begin{aligned}
& E_{X_{1}}^{\mathrm{int}}-E_{X_{1}}^{(0)} \approx-\mu_{1} \Lambda_{1} A_{2}, \\
& E_{X_{2}}^{\mathrm{int}}-E_{X_{2}}^{(0)} \approx-\mu_{2} \Lambda_{2} A_{1},
\end{aligned}
$$


where we introduced

$\mu_{1} \equiv \frac{\left[\alpha_{1}\left(1+\alpha_{2}\right)+\gamma_{1} \gamma_{2}\right] E_{X_{1}}^{(0)}+\gamma_{1} E_{X_{2}}^{(0)}}{\Lambda_{1} A_{2}\left[\left(1+\alpha_{1}\right)\left(1+\alpha_{2}\right)+\gamma_{1} \gamma_{2}\right]}$,

$\mu_{2} \equiv \frac{\left[\alpha_{2}\left(1+\alpha_{1}\right)+\gamma_{1} \gamma_{2}\right] E_{X_{2}}^{(0)}-\gamma_{2} E_{X_{1}}^{(0)}}{\Lambda_{2} A_{1}\left[\left(1+\alpha_{1}\right)\left(1+\alpha_{2}\right)+\gamma_{1} \gamma_{2}\right]}$,

we obtain the major partial potentials, $\Delta \Phi^{(i)}, i=1,2$, Eq. (19),

$$
\begin{aligned}
& \Delta \Phi^{(1)} \approx-\frac{\mu_{1} X_{1} A_{2} A_{1}^{3}}{2} \int_{0}^{\xi} \frac{d \xi}{\left(A_{1}^{2}+\xi\right) R_{\xi}}, \\
& \Delta \Phi^{(2)} \approx-\frac{\mu_{2} X_{2} A_{1} A_{2}^{3}}{2} \int_{0}^{\xi} \frac{d \xi}{\left(A_{2}^{2}+\xi\right) R_{\xi}},
\end{aligned}
$$

Recall that here $\xi\left(X_{1,2,3}\right)$ is the only positive root of cubic Eq. (18) and $R_{\xi}$ is defined by Eq. (20a). The total electrostatic potential outside the cloud is given by

$\Phi \approx-\sum_{i=1}^{3} E_{X_{i}}^{\mathrm{int}} X_{i}+\Delta \Phi^{(1)}+\Delta \Phi^{(2)}$,

where the internal-field components $E_{X_{i}}^{\mathrm{int}}$ are given by Eqs. (38) and (32).

Using Eq. (26), we obtain the major component of the induced electric field on the ionosphere side of the interface,

$\left.\Delta E_{X_{3}}\right|_{X_{3 B}} \approx \frac{\left(\mu_{1} X_{1}+\mu_{2} X_{2}\right) A_{3}}{X_{3 B}}$,

so that Eq. (28) gives the common value of the current density normal to the interface,

$\left.\left.J_{n}\right|_{X_{3 B}} \equiv J_{n}^{\mathrm{ext}}\right|_{X_{3 B}}=\left.K^{\mathrm{ext}} \Delta E_{X_{3}}\right|_{X_{3 B}}=\left.J_{n}^{\mathrm{int}}\right|_{X_{3 B}}$.

Similarly to Eq. (30), Eqs. (38) to (45) are valid where $X_{3 B} / A_{3}=\left(1-X_{1}^{2} A_{1}^{2}-X_{2}^{2} A_{2}^{2}\right)^{1 / 2} \gg A_{3} / A_{1,2}$. This covers the entire interface, except a narrow belt-like edge around the central plane $X_{3}=0$. Outside, the current density normal to the interface is mainly due to the parallel flow of electrons along $\boldsymbol{B}$. These electrons are collected into a semi-cloud, drift there, and return back to the ionosphere from the other semi-cloud, as shown in Fig. 2. The boundary plane between the two semi-clouds is determined by $\left.J_{n}\right|_{X_{3 B}}=0$, or

$\frac{X_{2}}{X_{1}}=-\frac{\mu_{1}}{\mu_{2}}$.

The internal current reaches its maximum value, $I_{\max }^{\text {int }}$, at this boundary. The current density within the cloud, $\boldsymbol{J}_{\boldsymbol{X}}^{\mathrm{int}}$, is uniform, so that the total internal current through a given cross-section is $\boldsymbol{J}_{\boldsymbol{X}}^{\mathrm{int}} \cdot \boldsymbol{S}$, where the $\boldsymbol{S}$ is the vector whose absolute value equals the cross-section area and where direction lies perpendicular to the cross-section plane. According Eqs. (33) and (41), we have

$J_{X_{1}}^{\text {int }}=\frac{K^{\mathrm{ext}} \mu_{1} A_{1}^{2}}{A_{3}}, \quad J_{X_{2}}^{\mathrm{int}}=\frac{K^{\mathrm{ext}} \mu_{2} A_{2}^{2}}{A_{3}}$ (and $J_{X_{3}}^{\text {int }}=0$, see above). The section along the plane described by Eq. (46) through the ellipsoid described by Eq. (12) is given by

$\boldsymbol{S}=\left[\begin{array}{c}S_{X_{1}} \\ S_{X_{2}} \\ S_{X_{3}}\end{array}\right]=\frac{\pi A_{1} A_{2} A_{3}}{\left(\mu_{2}^{2} A_{2}^{2}+\mu_{1}^{2} A_{1}^{2}\right)^{1 / 2}} \times\left[\begin{array}{c}\mu_{1} \\ \mu_{2} \\ 0\end{array}\right]$.

As a result, we obtain

$I_{\max }^{\mathrm{int}} \approx \pi K^{\mathrm{ext}} A_{1} A_{2}\left(\mu_{1}^{2} A_{1}^{2}+\mu_{2}^{2} A_{2}^{2}\right)^{1 / 2}$.

This current should coincide with the total ionospheric current $I_{\text {total }}^{\text {ext }}$ which is collected from the ionosphere into one semi-cloud (separated by Eq. 46) and released back from the other semi-cloud. We verify this in order to check the consistency of our mathematical treatment. According to Eqs. (27) and (45), the total collected and released current is given by

$I_{\mathrm{total}}^{\mathrm{ext}} \approx 2 K^{\mathrm{ext}} \iint \frac{\left(\mu_{1} X_{1}+\mu_{2} X_{2}\right) d X_{1} d X_{2}}{\left(1-X_{1}^{2} / A_{1}^{2}-X_{2}^{2} / A_{2}^{2}\right)^{1 / 2}}$,

where the double integration is carried out over either top or bottom side of one ellipsoid semi-cloud restricted by Eq. (46) and the factor 2 allows for the up-down symmetry of currents with respect to the central ellipsoidal plane, see Fig. 2. Near the central edge of the ellipsoid, the integrand is less accurate. Fortunately, its relative contribution to the entire integral is negligible, $\lesssim A_{3} / A_{1,2} \lll 1$. Calculating the integral in Eq. (50) via changing variables from $X_{1,2}$ to $\rho, \phi$, where $X_{1}=A_{1} \rho \cos \phi, X_{2}=A_{2} \rho \sin \phi$, with the integration domains of $0 \leq \rho \leq 1$ and $\phi_{0}=\arctan \left[-A_{1} \mu_{1} /\left(A_{2} \mu_{2}\right)\right] \leq \phi$ $\leq \phi_{0}+\pi$, we can verify that indeed $I_{\text {total }}^{\mathrm{ext}}=I_{\mathrm{max}}^{\mathrm{int}}$.

Equations (32), (38), (42-44), with parameters defined by Eqs. (10), (20), (34), (35), and (41), present the general analytic solution for the electric potentials (fields), both inside and outside the oblate ellipsoidal plasma cloud embedded in a homogeneous ionosphere with an external electric field. Equations (47) and (49), with $K^{\text {ext }}$ defined by Eq. (29), describe the current density in the cloud and the corresponding maximum total current. Major features of this solution vary significantly depending upon the relations between the ellipsoidal radii, $A_{i}$, and the plasma density ratio, $n^{\text {int }} / n_{0}$. The next section discusses how these features change with varying $n^{\text {int }} / n_{0}$ in the limiting case of a round (disk-like) plasma cloud. Appendix B discusses the opposite limit of an extended (rod-like) cloud.

\section{Round cloud (sporadic-E patch)}

In this section, we consider a particular but important case of a round (disk-like) cloud, $a \equiv a_{1}=a_{2}$, embedded in a vertical magnetic field, $\theta=90^{\circ}$. This particular case is representative for sporadic-E patches at high latitudes.

For this case, one could develop a simpler mathematical treatment from scratch, but in order to avoid repetition, 
we will use the general expressions obtained in the previous sections. For the vertical magnetic field, the renormalized coordinates in the horizontal plane, $X_{1,2}=x_{1,2}$, and $X_{3}=\epsilon x_{3}$. In this case, $E_{X_{1,2}}=E_{1,2}$, while $E_{X_{3}}=E_{3} / \epsilon$. Because a round pancake embedded in the vertical magnetic field has a rotational symmetry, we can align the $x_{1}$-axis along the external electric field, so that $E_{1}^{(0)}=E^{(0)}$, where $E^{(0)}$ is the external-field magnitude, and $E_{2,3}^{(0)}=0$. Additionally, we have for the parameters listed in previous sections, $\chi, p, \eta=0, A_{1}=A_{2}=a, A_{3}=\epsilon a_{3}, \Lambda_{1}=\Lambda_{2} \approx \pi / 4$,

$\alpha_{1}=\alpha_{2} \equiv \alpha \approx \frac{\pi \epsilon a_{3} n^{\mathrm{int}}}{4 a n_{0}}$,

$\gamma_{1}=\gamma_{2}=q \alpha \approx \frac{\pi a_{3} n^{\mathrm{int}}}{4 a n_{0}}\left(\frac{\psi}{1+\psi}\right)^{1 / 2}$,

where $q=\sigma_{\mathrm{H}} / \sigma_{\mathrm{P}}=\sqrt{\psi} /\left[(1+\psi) \Theta_{0}\right] \gg 1$, as defined in Eq. (A6b).

First, we discuss the electric field solution outside the cloud. Unlike the general ellipsoidal cloud, the disk-like interface allows us to express $\xi$ and $\Delta \Phi$ as simple functions of $X_{i}$. In this case, cubic Eq. (18) reduces to a quadratic equation with the only appropriate root given by

$\xi=\frac{R^{2}-A^{2}+\sqrt{\left(R^{2}-A^{2}\right)^{2}+4\left[\left(R^{2}-a^{2}\right) A_{3}^{2}+X_{3}^{2}\left(a^{2}-A_{3}^{2}\right)\right]}}{2}$,

where $R^{2}=\sum_{k=1}^{3} X_{k}^{2}=x_{1}^{2}+x_{2}^{2}+\epsilon^{2} x_{3}^{2}, A^{2}=a^{2}+\epsilon^{2} a_{3}^{2} \approx$ $a^{2}$. Not too close to the boundary surface, $\xi \gg \epsilon^{2} a_{3}^{2}$, we can also neglect $A_{3}^{2}$ in the expression for $R_{\xi}$, Eq. (20a), so that the total potential outside the cloud, Eq. (43), becomes

$$
\begin{aligned}
\Phi \approx & -\sum_{i=1}^{2} E_{i}^{\mathrm{int}} x_{i}+\frac{2 \sum_{i=1}^{2}\left(E_{x_{i}}^{\mathrm{int}}-E_{x_{i}}^{(0)}\right) x_{i}}{\pi} \\
& \times\left(\frac{\sqrt{\xi} / a}{1+\xi / a^{2}}+\arctan \frac{\sqrt{\xi}}{a}\right)
\end{aligned}
$$

where $E_{X_{i}}^{\text {int }} \approx E_{X_{i}}^{(0)}-\pi \mu_{i} a / 4$ and we imply the principal value of arctan. Here Eq. (41) reduces to

$$
\begin{aligned}
& \mu_{1} \approx \frac{4 \alpha\left(1+\alpha+q^{2} \alpha\right) E^{(0)}}{\pi a\left[(1+\alpha)^{2}+q^{2} \alpha^{2}\right]}, \\
& \mu_{2} \approx-\frac{4 \alpha q E^{(0)}}{\pi a\left[(1+\alpha)^{2}+q^{2} \alpha^{2}\right]} .
\end{aligned}
$$

Recall that the ratio of $\mu_{1}$ and $\mu_{2}$ determines the boundary between the inflowing and outflowing currents, as shown in Eq. (46). This approximate potential given by Eq. (53) satisfies the exact boundary conditions: $\left.\Phi\right|_{\xi \rightarrow \infty} \rightarrow$ $-\sum_{i=1}^{2} E_{i}^{(0)} x_{i}$ and $\left.\Phi\right|_{\xi=0}=-\sum_{i=1}^{2} E_{i}^{\text {int }} x_{i}$, so that one can disregard the small- $\xi$ restriction, $\xi \gg \epsilon^{2} a_{3}^{2}$, and apply Eq. (53) to all locations outside the cloud.

Next, we discuss the uniform fields and currents within the cloud. According to Eq. (38), we have $E_{3}^{\text {int }}=0$, while

$$
\begin{aligned}
& E_{1}^{\mathrm{int}} \approx \frac{(1+\alpha) E^{(0)}}{(1+\alpha)^{2}+q^{2} \alpha^{2}}, \\
& E_{2}^{\mathrm{int}} \approx \frac{q \alpha E^{(0)}}{(1+\alpha)^{2}+q^{2} \alpha^{2}} .
\end{aligned}
$$

From Eq. (55), the magnitude of the internal electric field, $E^{\text {int }} \equiv\left[\left(E_{1}^{\text {int }}\right)^{2}+\left(E_{2}^{\text {int }}\right)^{2}\right]^{1 / 2}$, equals

$$
E^{\text {int }} \approx \frac{E^{(0)}}{\left[(1+\alpha)^{2}+q^{2} \alpha^{2}\right]^{1 / 2}}
$$

This expression shows that the total internal electric field within the round cloud is always less than the external electric field. This distinguishes the disk-like cloud from the extended cloud discussed in Appendix B. The angle between $\boldsymbol{E}^{\text {int }}$ and $\boldsymbol{E}^{(0)}$, Eq. (39), is determined by

$\left|\tan \phi_{E}\right| \approx \frac{q \alpha}{1+\alpha}$.

Further, according to Eqs. (47), the internal current density in the original coordinates, $x_{1,2}$, is given by $J_{3}^{\text {int }}=0$ and

$J_{1}^{\mathrm{int}}=\frac{K^{\mathrm{ext}} \mu_{1} a^{2}}{a_{3}}, \quad J_{2}^{\mathrm{int}}=\frac{K^{\mathrm{ext}} \mu_{2} a^{2}}{a_{3}}$,

where $K^{\text {ext }}$ is defined by Eq. (29). The maximum total current, $I_{\max }^{\text {int }}$, across the vertical cross-section that separates the inflowing ionosphere currents from the outflowing ones, as depicted in Fig. 2, is given by Eq. (49) where

$$
\left(\mu_{1}^{2} A_{1}^{2}+\mu_{2}^{2} A_{2}^{2}\right)^{1 / 2} \approx \frac{4 \alpha\left(1+q^{2}\right)^{1 / 2} E^{\mathrm{int}}}{\pi\left[(1+\alpha)^{2}+q^{2} \alpha^{2}\right]^{1 / 2}},
$$

and we can further approximate the large factor in the numerator of Eq. (59), $\left(1+q^{2}\right)^{1 / 2} \approx q+(2 q)^{-1}$, by merely $q$. Expressing also $\alpha$ in the numerator of Eq. (59) through Eq. (51a) with $n^{\text {int }} / n_{0}=K^{\text {int }} / K^{\text {ext }}$, we obtain

$I_{\max }^{\mathrm{int}} \approx \frac{\pi \epsilon a a_{3} q K^{\mathrm{int}} E^{(0)}}{\left[(1+\alpha)^{2}+q^{2} \alpha^{2}\right]^{1 / 2}}$.

Using Eq. (A6) for $n=n^{\text {int }}$, we can express $I_{\max }^{\text {int }}$ in terms of the Hall conductivity within the cloud, $\sigma_{\mathrm{H}}^{\text {int }} \approx e n^{\text {int }} / B$, as

$I_{\max }^{\mathrm{int}} \approx \frac{\pi a a_{3} \sigma_{\mathrm{H}}^{\mathrm{int}} E^{(0)}}{\left[(1+\alpha)^{2}+q^{2} \alpha^{2}\right]^{1 / 2}}$.

We will use either Eq. (60a) or (60b) for qualitative interpretation in each of the two limiting cases discussed below. 


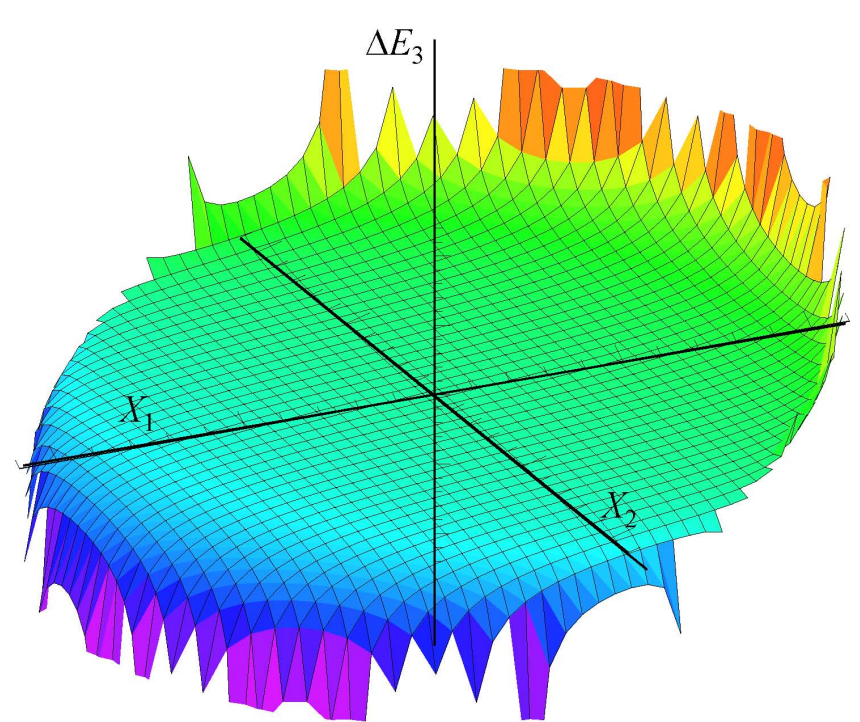

Fig. 3. Spatial distribution of $\left.\left.\Delta E_{3}\right|_{x_{3}} \propto J_{n}\right|_{x_{3 B}}$ (vertical axis). The thick line shows the divider between the inflowing and outflowing ionospheric currents in the $X_{1} X_{2}$-plane, Eq. (46).

Introducing dimensionless polar coordinates $\rho$ and $\phi_{x}$ via $x_{1}=\rho a \cos \phi_{x}, x_{2}=\rho a \sin \phi_{x}$, we obtain from Eq. (44) the parallel electric field on the ionosphere side of the ellipsoid boundary,

$\left.\Delta E_{3}\right|_{x_{3 B}} \approx \frac{4 \epsilon q \alpha \rho E^{(0)} \cos \left(\phi_{x}+\phi_{J}\right)}{\pi\left[(1+\alpha)^{2}+q^{2} \alpha^{2}\right]^{1 / 2}\left(1-\rho^{2}\right)^{1 / 2}}$,

where $\rho^{2}=\left(x_{1}^{2}+x_{2}^{2}\right) / a^{2}$ and

$\tan \phi_{J}=\frac{q}{1+\alpha\left(1+q^{2}\right)}$.

Figure 3 shows an example of the spatial distribution of $\left.\Delta E_{3}\right|_{x_{3}}$ over a cloud. The densities of the ionospheric currents that flow through the ionosphere-cloud interface, $\left.J_{n}\right|_{x_{3 B}}$, are proportional to $\left.\Delta E_{3}\right|_{x_{3}}$, and have the same spatial distribution. The parallel electric field goes to zero at the dividing plane defined by Eq. (46) and formally grows to infinity when approaching the cloud edge $\rho=1$. This is, however, valid only for $\left(1-\rho^{2}\right)^{1 / 2} \gg \epsilon a_{3} / a$. In the narrow belt-like area around $x_{3}=0$ where this restriction is lifted, the field actually saturates at a large but finite value corresponding to a very small factor in the denominator of the RHS of Eq. (61), namely, $\left(1-\rho^{2}\right)^{1 / 2} \sim \epsilon a_{3} / a$.

Now we discuss the dependence of the fields and currents on the plasma density ratio $n_{0} / n^{\text {int }}$. This dependence shows up via the parameter $\alpha$ in Eq. (51a). Since the latter is essentially a ratio of two small parameters, $\epsilon a_{3} / a$ and $n_{0} / n^{\text {int }}$, it spans a broad range from small to large values. Before going any further, we note the following. Because at the altitudes of interest the parameter $q^{2}$ is large, then for both large and small values of $\alpha$, one can approximate expressions like $(1+\alpha)^{2}+q^{2} \alpha^{2}$ by just $1+q^{2} \alpha^{2}$. This or similar approximations can be employed in simple estimates as valid interpolations for all values of $\alpha$, so that in all the above relations that involve such expressions depend largely on the value of the product $q \alpha$, rather than on $q$ and $\alpha$ separately.

We discuss now two opposite limits of small and large $q \alpha$. For a low-density cloud where

$q \alpha=\frac{\pi a_{3} n^{\mathrm{int}}}{4 a n_{0}}\left(\frac{\psi}{1+\psi}\right)^{1 / 2} \ll 1$,

we have, using Eq. (60b),

$\phi_{E} \approx \frac{q \alpha}{\pi} \times 180^{\circ} \ll 90^{\circ}, \quad E^{\mathrm{int}} \approx E^{(0)}$,

$I_{\max }^{\mathrm{int}} \approx \pi a a_{3} \sigma_{\mathrm{H}}^{\mathrm{int}} E^{(0)}$,

$\left.\Delta E_{3}\right|_{x_{3 B}} \approx \frac{4 \epsilon q \alpha \rho E^{(0)} \cos \left(\phi_{x}+\phi_{J}\right)}{\pi(1+\alpha)\left(1-\rho^{2}\right)^{1 / 2}}$.

In this limit, the induced field is relatively small, while the external field mostly penetrates into the cloud. As a result, the maximum current through the cloud in Eq. (64) depends largely on this field and the cloud plasma density, $n^{\mathrm{int}} \approx B \sigma_{\mathrm{H}}^{\mathrm{int}} / e$, while it is virtually independent of the ionosphere density, $n_{0}$. The maximum current through the cloud in this case is mostly the Hall current density caused by the external field, $\sigma_{\mathrm{H}}^{\text {int }} E^{(0)}$, multiplied by the maximum horizontal cross-section of the cloud, $\pi a a_{3}$.

This limit of small $q \alpha$ has a simple physical meaning. Although the cloud density is much larger that that of the ambient ionosphere, the latter still has enough charge carriers to close the current driven within the cloud by the nearly unaltered external electric field. Charge polarization at the sharp cloud edges, as well as small charge separation in the ambient ionosphere, create additional polarization fields which are small compared to the external field, but sufficient to sustain the required ionospheric current closure. This explanation suggests that the regime when the external field mostly penetrates into the cloud should take place for rather generally shaped clouds with the non-homogeneously distributed plasma density. In such cases, the internal current is simply determined by the cloud conductivity, while the configuration of the closing ionospheric currents requires a solution of Eq. (3) for the specific cloud geometry and plasma density distribution.

For higher density ratio, $n_{0} / n^{\text {int }}$, corresponding to $q \alpha \sim 1$, we have general Eqs. (56) to (62), where we must retain $q^{2} \alpha^{2} \sim 1$ but, due to $q^{2} \gg 1$, can neglect $\alpha$ which in this case is small in comparison with unity. Figure 4 illustrates this intermediate case. It shows the spatial distribution of the electrostatic potential in the near-cloud region. The electric fields are perpendicular to the equipotential contours shown by solid curves. Qualitatively, such distribution is typical 


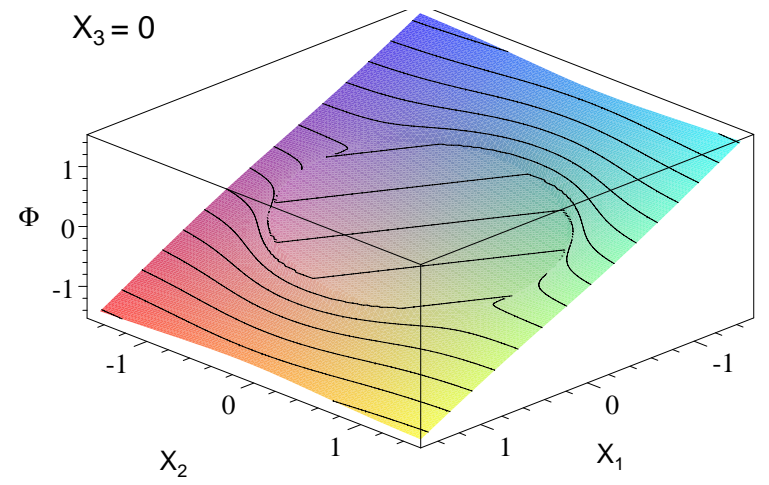

(a)

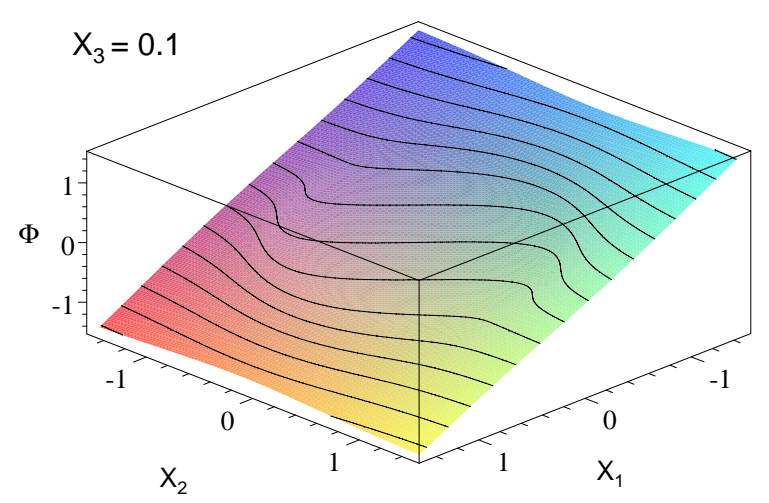

(c)

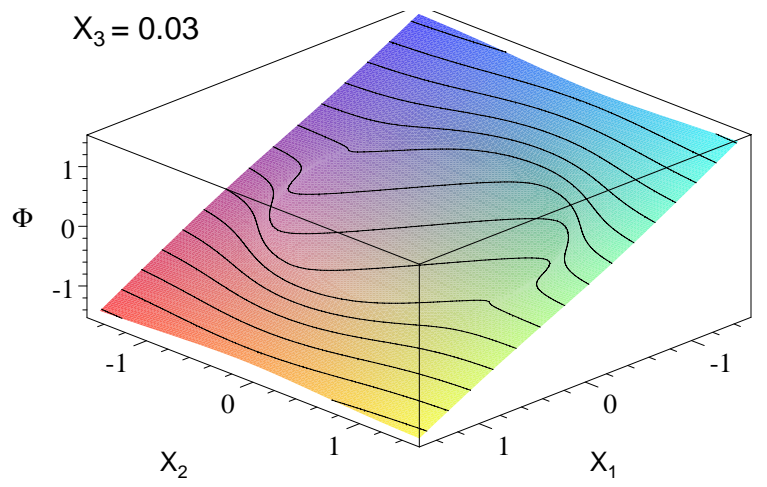

(b)

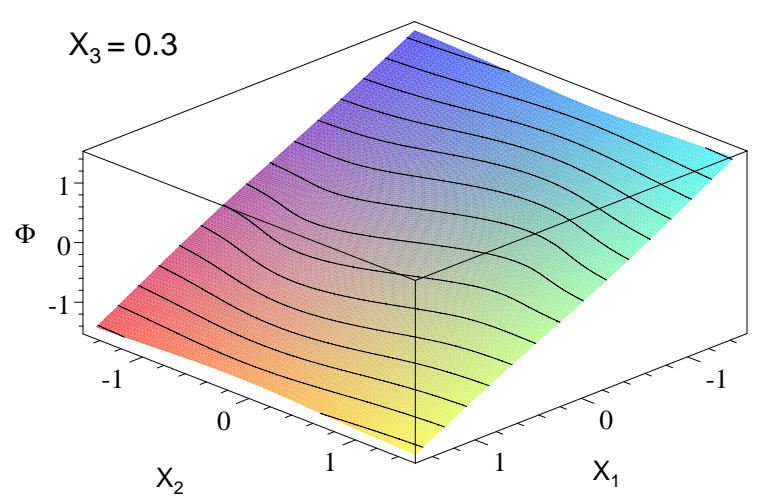

(d)

Fig. 4. Distribution of electrostatic potential in horizontal cross-sections of the near-cloud region by four different vertical distances in $X_{3}$ coordinates (the corresponding values of $X_{3}$ are shown in the upper-left corner of each figure). The solid curves show equipotential contours. Here $\alpha=0.1, q=20$ (corresponding to $\psi \simeq 8.6 \times 10^{-2}$ ), so that $q \alpha=2$. Further, $a=1$ and the external electric field is directed along $X_{1}$ : $E_{X_{1}}^{(0)}=1, E_{X_{2}}^{(0)}=0$. The central circular area of $X_{1}^{2}+X_{2}^{2}=1$ corresponds to the position of the disk-like cloud at $X_{3}=0$. The boundary between cross-sections intersecting the cloud and those that lie outside it corresponds to $X_{3}=\epsilon a_{3}$, where $\epsilon a_{3}=0.01$ is the cloud radius in a vertical direction. Only in (a) the cross-section intersects the cloud; in all three other cases, the cross-sections lie fully outside the cloud. Notice, however, a close similarity between the potentials in (a) and (b).

for any clouds, although the characteristic scales may differ significantly. Panel (a) shows the horizontal distribution of the potential in the central plane that intersects the cloud. Within the cloud area, one can clearly see the internal electric field rotated with respect to the external field. Outside the cloud near its boundary, one can see a sharp transition to a potential which approaches the linearly dependent potential corresponding to the external electric field at a rather short horizontal distance from the cloud. It is interesting that essentially the same potential distribution, but without a smoothen transition, takes place even well beyond the cloud (see panel (b) corresponding to $X_{3}=0.03>\epsilon \alpha_{3}=0.01$ ). At a larger vertical distance from the cloud, $X_{3}=0.1$ (c), the internal field still clearly differs from the external field, both in the magnitude and direction. However, as $X_{3}$ increases further, $X_{3}=0.3(\mathrm{~d})$, the effect of the cloud gradu- ally disappears. The spatial distribution of the potential is virtually insensitive to the smallest (vertical) cloud size; for any oblate clouds, $A_{3} \ll a$, the corresponding figures look essentially indistinguishable from Fig. 4. This is, however, only true if we are not interested in the near-cloud vertical electric field, $\Delta E_{X_{3}}$, and currents; the two may depend strongly upon the vertical cloud size. The vertical distances over which the cloud effects disappear are comparable to the horizontal cloud size, $a=1$ (but less than 1). These distances characterize the effective size of the near-cloud region where spatial uniformity of the background ionosphere density and external electric field, which are not included in this model, can affect the entire electrodynamic structure. Beyond this region, all inhomogeneities, including other clouds, F region, etc., should not exert a significant influence on the near-cloud fields and currents. Note that the configurations 
of the internal field and current in the intermediate case of $q \alpha \sim 1$ are more sensitive to the specific choice of the cloud geometry than in the case of $q \alpha \ll 1$.

As the density ratio $n_{0} / n^{\text {int }}$ grows further, we pass to the opposite limit of

$q \alpha=\frac{\pi a_{3} n^{\mathrm{int}}}{4 a n_{0}}\left(\frac{\psi}{1+\psi}\right)^{1 / 2} \gg 1$,

where, according to Eqs. (60a) and (A6a),

$$
\begin{aligned}
\phi_{E} & \approx 90^{\circ}, \quad E^{\mathrm{int}} \approx E^{(0)} /(q \alpha) \ll E^{(0)}, \\
I_{\max }^{\mathrm{int}} \approx 4 a^{2} \sigma_{\|}^{\mathrm{ext}} \in E^{(0)} & \approx \frac{4 a^{2} e n^{\mathrm{ext}} E^{(0)}}{B}\left(\frac{1+\psi}{\psi}\right)^{1 / 2} .
\end{aligned}
$$

$\left.\Delta E_{3}\right|_{x_{3 B}} \approx \frac{4 \epsilon \rho E^{(0)} \cos \left(\phi_{x}+\phi_{J}\right)}{\pi\left(1-\rho^{2}\right)^{1 / 2}}$.

In this limit, the internal field becomes much less in magnitude than the external field and lies practically orthogonal to the latter. Because the cloud is nearly equipotential, the induced field outside it reaches its maximum possible value. We see that at $\phi_{x} \simeq-\phi_{J}$ and, especially, on approaching the cloud edge on the margin of Eq. (61) applicability, $\left(1-\rho^{2}\right)^{1 / 2} \sim \epsilon a_{3} / a$, the induced electric field parallel to $\boldsymbol{B}$ can reach large values $\left.\Delta E_{3}\right|_{x_{3} B} \sim a E^{(0)} / a_{3} \gg E^{(0)}$. Even a moderate external field of $E^{(0)} \sim 1 \mathrm{mV} / \mathrm{m}$ and $a / a_{3} \sim 100$, will generate near the cloud edges parallel electric fields of $\left.\Delta E_{3}\right|_{x_{3 B}} \sim 100 \mathrm{mV} / \mathrm{m}$, which can raise the electron temperature significantly (Dimant et al., 2009). At midlatitudes, the electric field component perpendicular to $\boldsymbol{B}$ should also increase. However, this does not necessarily mean a proportional improvement in the conditions required for generation of E-region instabilities because the elevated electron temperature raises the instability threshold. A more accurate quantitative prediction requires a self-consistent inclusion of strong local electron heating into the model, which is beyond the framework of this paper. Note also that the estimated field of around $100 \mathrm{mV} / \mathrm{m}$ should be a rare event because for large clouds it is not so easy to reach the limit required by Eq. (66), as we discuss below. One might expect that near-cloud fields will typically reach a few tens of $\mathrm{mV} / \mathrm{m}$.

In the limiting case of Eq. (66), the total current in the cloud, $I_{\max }^{\mathrm{int}}$, is determined almost entirely by the charge collection from the ionosphere and is virtually independent of the cloud plasma density. The total collection current from the ionosphere, $I_{\text {total }}^{\mathrm{ext}}=I_{\max }^{\mathrm{int}}$, is proportional to the ionospheric density and is mainly caused by electrons drifting along $\boldsymbol{B}$. On most of the cloud interface, this electron drift is a response to the induced electric field $\Delta E_{3} \sim E^{(0)}$, which is nearly parallel to $\boldsymbol{B}$. The maximum cloud current determined by Eq. (67) is a saturation current reached when the external field is completely expelled from the cloud. The saturated current, $I_{\mathrm{sat}}^{\text {cloud }}=I_{\max }^{\mathrm{int}}(q \alpha \gg 1)$, can be written in practical units as

$I_{\text {sat }}^{\text {cloud }} \approx 12.8 \mathrm{kA}\left(\frac{1+\psi}{\psi}\right)^{1 / 2}\left(\frac{a}{100 \mathrm{~km}}\right)^{2}\left(\frac{E^{(0)}}{1 \mathrm{mV} / \mathrm{m}}\right)\left(\frac{5 \times 10^{4} \mathrm{nT}}{B}\right)$.

For typical sporadic-E layers, $a \gtrsim 100 \mathrm{~km}$, and moderate values of the external field, $E^{(0)} \gtrsim 1 \mathrm{mV} / \mathrm{m}$, the saturated current through the cloud can reach tens of kiloamperes, comparable to the electrojet currents. However, the larger the cloud horizontal radius $a$ (compared to its vertical thickness, $a_{3}$ ), the more difficult it is to reach the dense-cloud limit of $q \alpha \gg 1$. Indeed, according to Eq. (51b), if we have $a_{3} \simeq 1 \mathrm{~km}, a \simeq 100 \mathrm{~km}, \psi \simeq 0.1$ then, in order to reach this limit, we need a density ratio of $n^{\text {int }} / n_{0} \gg 4 \times 10^{2}$. For lower-density sporadic-E layers, $n^{\text {int }} \sim 10^{11-12} \mathrm{~m}^{-3}$, under nighttime ionosphere conditions, $n_{0} \lesssim 10^{10} \mathrm{~m}^{-3}$, this limit is barely reachable. Nevertheless, even in a more realistic case of $q \alpha \sim 1$, the total current through one or more clouds can be quite significant and affect the entire ionospheric conductance, as we discuss at the end of this section.

For non-zero $\theta$ and a non-round cloud, analytic expressions are more complicated but, for comparable $a_{1}$ and $a_{2}$, the major results are qualitatively the same. An important result is that there should be no amplification of the electric field inside the cloud, mainly its rotation and reduction. However, the field can increase significantly outside the cloud. This has an important implication for excitation of Farley-Buneman and other E-region instabilities at midlatitudes (Haldoupis et al., 1996).

The strongest induced electric field outside the cloud is near the cloud interface, where it is perpendicular to it. Only the field component perpendicular to $\boldsymbol{B}$ can drive instabilities, while the parallel component can only heat electrons. At first glance, this mechanism of instability generation seems to be more relevant for midlatitudes where the magnetic field is far from vertical. However, at the high-latitude electrojet, especially during strong magnetospheric perturbations, small $\left(90^{\circ}-\theta\right)$ can be compensated by much higher external electric fields.

To conclude this section, we now calculate effective ionosphere conductances associated with currents that flow through a sporadic-E cloud. These conductances can be added to the conventional conductance models used for magnetosphere-ionosphere coupling in global MHD simulations of the magnetosphere (e.g., Fedder et al., 1995; Raeder et al., 2001; Ridley et al., 2004). Some modifications of the conventional conductances have been invoked, e.g., to reconcile disagreements of model results with observations of cross-cap potentials (Merkin et al., 2005). The inclusion of sporadic-E layer conductances are of special importance for nighttime side of the high-latitude ionosphere where, in existing models, only conductance modifications associated with particle precipitation have been taken into account. 
The conventional global ionospheric conductance tensor has a simple structure with only two independent parameters, namely, the Pedersen and Hall conductances, $\Sigma_{\mathrm{P}, \mathrm{H}}$. As this paper demonstrates, sporadic-E clouds can easily be polarized, and the corresponding effective conductance tensors in the general case have a more complicated structure which depends on the cloud orientation. However, for the rotationally symmetric clouds in a nearly vertical magnetic field, the effective conductance tensor reduces to also just two independent conductances, $\Sigma_{\mathrm{P}, \mathrm{H}}^{\mathrm{eff}}$ similar to $\Sigma_{\mathrm{P}, \mathrm{H}}$.

In global MHD simulations, the entire ionosphere represents a thin boundary layer with given conductances. This implies that the electric field inside it is uniform, mapped from the magnetosphere. Such an approach disregards local electric-field inhomogeneities caused by polarization of sporadic-E clouds. To calculate the effective Pedersen and Hall conductances, $\Sigma_{\mathrm{P}, \mathrm{H}}^{\mathrm{eff}}$, to be included into global MHD codes, we need to integrate the corresponding components of the current density over the vertical thickness of the cloud and divide them by the external electric field magnitude, $\Sigma_{\mathrm{P}}^{\mathrm{eff}}=\int J_{1}^{\mathrm{int}} d x_{3} / E^{(0)}$ and $\Sigma_{\mathrm{H}}^{\mathrm{eff}}=\int J_{2}^{\mathrm{int}} d x_{3} / E^{(0)}$. Here the external electric field $E^{(0)}$ approximates the global magnetospheric field. Because the current densities given by Eq. (58) are uniform, the integration over altitudes within the cloud, at a given horizontal location determined by the coordinates $x_{1,2}$, reduces to a simple multiplication by the corresponding cloud thickness, $2 x_{3 B}\left(x_{1,2}\right)=2 a_{3}\left[1-\left(x_{1}^{2}+x_{2}^{2}\right) / a^{2}\right]^{1 / 2}$. Using Eqs. (58) with $\mu_{1,2}$ defined by Eq. (54), where $\alpha$ in the numerators is expressed by Eq. (51a) and $K^{\text {ext }}$ expressed as $\left(n_{0} / n^{\text {int }}\right) \sigma_{\mathrm{P}}^{\text {int }} / \epsilon$, we obtain

$\Sigma_{\mathrm{P}}^{\mathrm{eff}}=\frac{2\left(1+\alpha+q^{2} \alpha\right) a_{3} \sigma_{\mathrm{P}}^{\mathrm{int}}}{(1+\alpha)^{2}+q^{2} \alpha^{2}}\left(1-\frac{x_{1}^{2}+x_{2}^{2}}{a^{2}}\right)^{1 / 2}$,

$\Sigma_{\mathrm{H}}^{\mathrm{eff}}=\frac{2 a_{3} \sigma_{\mathrm{H}}^{\mathrm{int}}}{(1+\alpha)^{2}+q^{2} \alpha^{2}}\left(1-\frac{x_{1}^{2}+x_{2}^{2}}{a^{2}}\right)^{1 / 2}$.

Here, for magnetized electrons and unmagnetized ions, $\sigma_{\mathrm{P}}^{\text {int }} \approx(1+\psi) \Theta_{0} n^{\text {int }} e /(B \sqrt{\psi})$ and $\sigma_{\mathrm{H}}^{\text {int }} \approx n^{\text {int }} e / B$, as given by Eq. (5). Note that the multipliers $2 a_{3} \sigma_{\mathrm{P}, \mathrm{H}}^{\mathrm{int}}\left[1-\left(x_{1}^{2}+\right.\right.$ $\left.\left.x_{2}^{2}\right) / a^{2}\right]^{1 / 2}$ in Eq. (70) describe conductances associated with the increased number of charged carriers within the cloud, while the additional factors describe the effects of cloud polarization. For any values of $\alpha$, in accord with simple interpolation estimates discussed above, we can approximate $1+\alpha+q^{2} \alpha$ by $1+q^{2} \alpha$, so that the ratio of the two effective conductances becomes

$\frac{\Sigma_{\mathrm{P}}^{\mathrm{eff}}}{\Sigma_{\mathrm{H}}^{\mathrm{eff}}} \simeq \frac{1}{q}+q \alpha=\frac{\Sigma_{\mathrm{P}}}{\Sigma_{\mathrm{H}}}+q \alpha$.

This ratio remains the same as for the regular ionosphere conductances, $\Sigma_{\mathrm{P}}^{\text {eff }} / \Sigma_{\mathrm{H}}^{\mathrm{eff}} \simeq \Sigma_{\mathrm{P}} / \Sigma_{\mathrm{H}} \ll 1$ only in the limit of very small $q \alpha$, when $q^{2} \alpha \ll 1$. In this case, $\Sigma_{\mathrm{H}}^{\text {eff }} \approx$

$$
\begin{aligned}
& \Sigma_{\mathrm{H}_{\text {max }}}^{\text {eff }}\left[1-\left(x_{1}^{2}+x_{2}^{2}\right) / a^{2}\right]^{1 / 2}, \text { where } \\
& \Sigma_{\mathrm{H}_{\text {max }}}^{\text {eff }} \equiv 2 a_{3} \sigma_{\mathrm{H}}^{\mathrm{int}} \approx 6.4\left(\frac{n^{\mathrm{int}}}{10^{12} \mathrm{~m}^{-3}}\right)\left(\frac{5 \times 10^{4} \mathrm{nT}}{B}\right)\left(\frac{a_{3}}{1 \mathrm{~km}}\right) \text { mho, }
\end{aligned}
$$

because in this limit the external field mostly penetrates into the cloud, as described by Eq. (64).

In the opposite limit of large $q \alpha$, Eq. (66), the cloud polarization plays a crucial role. In this case, the effective Pedersen conductance can exceed significantly the effective Hall conductance, $\Sigma_{\mathrm{P}}^{\text {eff }} / \Sigma_{\mathrm{H}}^{\text {eff }} \simeq q \alpha \gg 1$. This is of paramount importance for global magnetospheric modeling, because makes the ionospheric current closure more efficient. We should bear in mind, however, that in this case the largest conductance, $\Sigma_{\mathrm{P}}^{\text {eff }}$, can differ noticeably from that calculated without the effect of cloud polarization,

$$
\begin{aligned}
\Sigma_{\mathrm{P}}^{\mathrm{eff}} & \simeq \frac{2 a_{3} \sigma_{\mathrm{P}}^{\mathrm{int}}}{\alpha}\left(1-\frac{x_{1}^{2}+x_{2}^{2}}{a^{2}}\right)^{1 / 2} \\
& =\frac{2 a_{3} \sigma_{\mathrm{H}}^{\mathrm{int}}}{q \alpha}\left(1-\frac{x_{1}^{2}+x_{2}^{2}}{a^{2}}\right)^{1 / 2} \ll 2 a_{3} \sigma_{\mathrm{H}}^{\mathrm{int}} .
\end{aligned}
$$

In the intermediate case of $q \alpha \sim 1$, we approximate Eq. (70) by

$$
\begin{aligned}
& \Sigma_{\mathrm{P}}^{\mathrm{eff}} \simeq \frac{2 q \alpha a_{3} \sigma_{\mathrm{H}}^{\mathrm{int}}}{1+q^{2} \alpha^{2}}\left(1-\frac{x_{1}^{2}+x_{2}^{2}}{a^{2}}\right)^{1 / 2}, \\
& \Sigma_{\mathrm{H}}^{\mathrm{eff}} \simeq \frac{2 a_{3} \sigma_{\mathrm{H}}^{\mathrm{int}}}{1+q^{2} \alpha^{2}}\left(1-\frac{x_{1}^{2}+x_{2}^{2}}{a^{2}}\right)^{1 / 2},
\end{aligned}
$$

so that the Pedersen conductance proves to be comparable to the Hall one, $\Sigma_{\mathrm{P}}^{\text {eff }} \simeq q \alpha \Sigma_{\mathrm{H}}^{\mathrm{eff}}$, reaching at most about a half of the maximum Hall conductance given by Eq. (72). For typical sporadic-E clouds with $a_{3} \sim 1 \mathrm{~km}$ and $n^{\text {int }} \sim 10^{12} \mathrm{~m}^{-3}$, $\Sigma_{\mathrm{P}}^{\text {eff }}$ can reach a few mho, which is comparable to typical daytime ionosphere conductances, $\Sigma_{\mathrm{P}, \mathrm{H}} \lesssim 20$ mho (Moen and Brekke, 1993). This means that the effect of sporadic-E clouds may play an important role in providing conductance in the nighttime high-latitude ionosphere and should be taken into account in global magnetosphere-ionosphere coupling models.

\section{Summary and conclusions}

In this paper, we have studied the 3-D electrodynamic interaction of an external electric field with a dense plasma cloud embedded in the lower-E/upper-D ionosphere. To make an analytic solution possible, we have employed a simplified physical model with the following major assumptions: (1) uniformly dense plasma cloud bounded from the background ionosphere by an oblate 3-D ellipsoid; (2) homogeneous and unbounded tenuous ionosphere; (3) uniform external electric field perpendicular to the magnetic field $\boldsymbol{B}$. Using 
this model, we have obtained the general analytic solution for the electric potential and currents in the entire space and have discussed limiting cases. While the actual situation is more complicated, our analytic solution gives an understanding of various regimes depending on crucial parameters and for quantitative estimates of the expected physical effects.

The major quantitative results of our analytic model can be summarized as follows. The 3-D electric field and current depend upon the relations between the ellipsoid semi-axes and the ratio of the plasma densities within the cloud and surrounding ionosphere. Outside the cloud, the electric field on the ionospheric side of the interface are described in the general case by Eqs. (42-44). This field lies perpendicular to the interface and can have a significant component parallel to $\boldsymbol{B}$. Within the ellipsoidal cloud, the uniform electric field and current density are described by Eqs. (32), (38), and (47). The internal electric field is rotated with respect to the external field, while its amplitude can be both larger or smaller that that of the external field. The maximum total current collected from the ionosphere in one semi-cloud and released from the other semi-cloud is given by Eq. (49). It can be quite significant, especially in the important particular case of a disk-like cloud, Sect. 5.

The latter model can serve as a reasonable approximation to actual sporadic-E layers. In this case, the internal electric field never exceeds the external field, while on the ionospheric side it can be larger. For low-density clouds, the external field penetrates into the cloud, so that the internal cloud current there is determined by the cloud conductivity. The induced electric field and collected ionospheric currents in this case are relatively small. For denser clouds, the internal electric field cannot easily penetrate, leaving an almost equipotential cloud. In this case, the total current through the cloud nearly reaches its saturation value determined by the ionospheric conductivity, Eqs. (67) and (69). The induced electric field on the ionospheric side of the ellipsoidal interface also reaches its maximum values, Eq. (68). All these effects are fundamentally three-dimensional. Estimates show that sporadic-E clouds can make noticeable local contributions to ionospheric conductances at nighttime side. This is of importance for modeling magnetosphere-ionosphere coupling in global MHD simulations used for Space Weather predictions.

This analytical model can be employed for simple estimates of near-cloud fields and currents for specific cloud sizes and average plasma parameters. More realistic studies will require including ionospheric and cloud-plasma inhomogeneities, as well as global layers of distinct ionospheric conductivities, temporal variability of fields and plasmas and simultaneous presence of several separate plasma patches. All these important factors seriously hinder analytical studies, but they could be included in future powerful computer simulations.

\section{Appendix A}

\section{Coordinate transformations}

In this Appendix, we make coordinate transformations that allow us to reduce Eq. (3) to Laplace's Eq. (16), while keeping the simple canonical form for the ellipsoidal plasma cloud. We express these linear transformations in matrix form, so that sequential coordinate transformations are described by multiplication of the corresponding matrices.

In order to efficiently apply Eq. (4), we need a coordinate system aligned with the magnetic field. To reach this, we rotate our original coordinates in such a way that the new axis $\boldsymbol{x}_{3}^{\prime}$ becomes directed along $\boldsymbol{B}, \boldsymbol{x}_{2}^{\prime}$ becomes perpendicular to axes $\boldsymbol{x}_{3}$ and $\boldsymbol{x}_{3}^{\prime}$, while the remaining axis $\boldsymbol{x}_{1}^{\prime}$ keeps its required perpendicularity to the new axes $\boldsymbol{x}_{2}^{\prime}$ and $\boldsymbol{x}_{3}^{\prime}$, see Fig. 1. This coordinate rotation, $x_{i}^{\prime}=\sum_{k=1}^{3}\left(\partial x_{i}^{\prime} / \partial x_{k}\right) x_{k}$, can be described by the transformation matrix

$$
\begin{aligned}
{\left[\frac{\partial x_{i}^{\prime}}{\partial x_{k}}\right] } & =\left[\begin{array}{lll}
\partial x_{1}^{\prime} / \partial x_{1} & \partial x_{1}^{\prime} / \partial x_{2} & \partial x_{1}^{\prime} / \partial x_{3} \\
\partial x_{2}^{\prime} / \partial x_{1} & \partial x_{2}^{\prime} / \partial x_{2} & \partial x_{2}^{\prime} / \partial x_{3} \\
\partial x_{3}^{\prime} / \partial x_{1} & \partial x_{3}^{\prime} / \partial x_{2} & \partial x_{3}^{\prime} / \partial x_{3}
\end{array}\right] \\
= & {\left[\begin{array}{ccc}
\sin \theta \cos \varphi & \sin \theta \sin \varphi & -\cos \theta \\
-\sin \varphi & \cos \varphi & 0 \\
\cos \theta \cos \varphi & \cos \theta \sin \varphi & \sin \theta
\end{array}\right], }
\end{aligned}
$$

acting on the three-component vector $\left[x_{k}\right]=\left[x_{1}, x_{2}, x_{3}\right]$. The inverse coordinate transformation, $x_{k}=\sum_{i=1}^{3}\left(\partial x_{k} / \partial x_{i}^{\prime}\right) x_{i}^{\prime}$, is described by the transposed matrix. Rotation is a unitary coordinate transformation, $\partial x_{k} / \partial x_{i}^{\prime}=\partial x_{i}^{\prime} / \partial x_{k}$ with $\operatorname{det}\left[\partial x_{i}^{\prime} / \partial x_{k}\right]=1$, so that the components of any covariant and contravariant vectors transform with the same matrix, $A_{x_{i}^{\prime}}=\sum_{k=1}^{3}\left(\partial x_{i}^{\prime} / \partial x_{k}\right) A_{x_{k}}$.

Equations (4) and (5) involve parallel and Pedersen conductivities with strongly different contributions to Eq. (3). To simplify this equation by making the contributions alike, we make our second coordinate transformation via rescaling the coordinate along $\boldsymbol{B}$,

$x_{3}^{\prime} \rightarrow X_{3}^{\prime} \equiv \epsilon x_{3}^{\prime}$,

while keeping invariant the two other coordinates, $X_{1,2}^{\prime} \equiv$ $x_{1,2}^{\prime}$. Here $\epsilon \approx \Theta_{0}(1+\psi)^{1 / 2}$, Eq. (7), is a small parameter because both $\Theta_{0}$, Eq. (6), and $\Theta_{0} \psi^{1 / 2}=v_{e n} / \Omega_{e}$ are small. This linear transformation, $X_{i}^{\prime}=\sum_{k=1}^{3}\left(\partial X_{i}^{\prime} / \partial x_{k}^{\prime}\right) x_{k}^{\prime}$, is described by a simple diagonal matrix

$\left[\frac{\partial X_{i}^{\prime}}{\partial x_{k}^{\prime}}\right]=\left[\begin{array}{lll}1 & 0 & 0 \\ 0 & 1 & 0 \\ 0 & 0 & \epsilon\end{array}\right]$.

The inverse transformation is described by the inverse matrix $\left[\partial X_{i}^{\prime} / \partial x_{k}^{\prime}\right]^{-1}=\left[\partial x_{k}^{\prime} / \partial X_{i}^{\prime}\right]$, which coincides with $\left[\partial X_{i}^{\prime} / \partial x_{k}^{\prime}\right]$, except for the last diagonal component, $\partial x_{3}^{\prime} / \partial X_{3}^{\prime}=1 / \epsilon$. Rescaling is not a unitary operation, 
so that covariant and contravariant vectors transform differently. Additionally, the plasma density changes: the plasma "density" in the $X_{k}^{\prime}$-coordinates, $N$, is related to the original plasma density, $n$, as $N / n=\operatorname{det}\left[\partial x_{k}^{\prime} / \partial X_{i}^{\prime}\right]=$ $1 / \epsilon$. The covariant electric field transforms according to $E_{X_{i}^{\prime}}=\sum_{k=1}^{3}\left(\partial x_{k}^{\prime} / \partial X_{i}^{\prime}\right) E_{x_{k}^{\prime}}$, while the current density transforms according to $J_{X_{i}^{\prime}}=(N / n) \sum_{k=1}^{3}\left(\partial X_{i}^{\prime} / \partial x_{k}^{\prime}\right) j_{x_{k}^{\prime}}=$ $(1 / \epsilon) \sum_{i=1}^{3}\left(\partial X_{k}^{\prime} / \partial x_{i}^{\prime}\right) j_{x_{k}^{\prime}}^{\prime}$, where $E_{X_{i}^{\prime}}, E_{x_{k}^{\prime}}$ and $J_{X_{i}^{\prime}}, j_{x_{k}^{\prime}}$ are the components of the electric field and current density in the $X_{k}^{\prime}$ - and $x_{k}^{\prime}$-coordinates, respectively. From these transformation rules, we obtain $J_{X_{1,2}^{\prime}}=j_{x_{1,2}^{\prime}}^{\prime} / \epsilon, J_{X_{3}^{\prime}}=j_{x_{3}^{\prime}}^{\prime}, E_{x_{1,2}^{\prime}}=$ $E_{X_{1,2}^{\prime}}, E_{x_{3}^{\prime}}=\epsilon E_{X_{3}^{\prime}}$, so that Eqs. (4) and (5) yield

$J_{X_{i}^{\prime}}=\sum_{k=1}^{3} \sigma_{X_{k}^{\prime}}^{X_{i}^{\prime}} E_{X_{K}^{\prime}}$.

with the new conductivity tensor given by

$\left[\begin{array}{c}X_{i}^{\prime} \\ \sigma_{X_{k}^{\prime}}^{\prime}\end{array}\right]=\left[\begin{array}{ccc}\sigma_{X_{1}^{\prime}}^{X_{1}^{\prime}} & \sigma_{X_{2}^{\prime}}^{X_{1}^{\prime}} & \sigma_{X_{3}^{\prime}}^{X_{1}^{\prime}} \\ \sigma_{X_{1}^{\prime}}^{X_{X_{1}^{\prime}}^{\prime}} & \sigma_{X_{2}^{\prime}}^{X_{2}^{\prime}} & \sigma_{X_{3}^{\prime}}^{X_{2}^{\prime}} \\ X_{3}^{\prime} & \sigma_{3}^{\prime} & X_{3}^{\prime} \\ \sigma_{X_{1}^{\prime}}^{\prime} & \sigma_{X_{2}^{\prime}} & \sigma_{X_{3}^{\prime}}^{\prime}\end{array}\right]=K \times\left[\begin{array}{ccc}1 & q & 0 \\ -q & 1 & 0 \\ 0 & 0 & 1\end{array}\right]$,

where

$$
\begin{aligned}
K & \equiv \frac{\sigma_{\mathrm{P}}}{\epsilon}=\epsilon \sigma_{\|}=\left(\frac{1+\psi}{\psi}\right)^{1 / 2} \frac{e n}{B}, \\
q & \equiv \frac{\sigma_{\mathrm{H}}}{\sigma_{\mathrm{P}}}=\frac{\sqrt{\psi}}{(1+\psi) \Theta_{0}} .
\end{aligned}
$$

Within the allowed range of $\psi$ determined by $v_{i} / \Omega_{i}=$ $\psi^{1 / 2} / \Theta_{0} \gg 1$ and $v_{e} / \Omega_{e}=\psi^{1 / 2} \Theta_{0} \ll 1$, i.e., for $\Theta_{0}^{2} \ll$ $\psi \ll \Theta_{0}^{-2}$, the parameter $q$ is large, but not too large, $q \leq$ $\left(2 \Theta_{0}\right)^{-1} \simeq 35$.

Anisotropic rescaling $x_{i}^{\prime} \rightarrow X_{i}^{\prime}$ leads to further effective flattening of the already oblate ellipsoid. In addition to that, the central ellipsoidal plane effectively rotates to become nearly perpendicular to $\boldsymbol{B}$. We complete the chain of coordinate transformations by a two-step 3-D rotation of the coordinate axes $\boldsymbol{X}_{k}^{\prime}$ to final axes $\boldsymbol{X}_{k}$, which are aligned with the principal axes of the anisotropically rescaled ellipsoid. The standard way do this is via matrix diagonalization (e.g., Riley et al., 1998). To avoid complicated equations associated with the standard technique, we use a simpler approximate approach. First, we rotate the coordinate system around the $\boldsymbol{X}_{2}^{\prime}$ through a small angle $\chi$ towards the original $\boldsymbol{x}_{3}$-axis in such a way that the central ellipsoid plane becomes perpendicular to the rotated $\boldsymbol{X}_{3}^{\prime}$-axis. The coordinate transformation from $X_{n}^{\prime}$ to the new intermediate coordinates $X_{m}^{\prime \prime}$, $X_{m}^{\prime \prime}=\sum_{n=1}^{3}\left(\partial X_{m}^{\prime \prime} / \partial X_{n}^{\prime}\right) X_{n}^{\prime}$ is described by the unitary matrix

$\left[\frac{\partial X_{m}^{\prime \prime}}{\partial X_{n}^{\prime}}\right]=\left[\begin{array}{ccc}\cos \chi & 0 & \sin \chi \\ 0 & 1 & 0 \\ -\sin \chi & 0 & \cos \chi\end{array}\right]$
The entire transformation from the original coordinates $x_{i}$ to $X_{m}^{\prime \prime}$ is described by the matrix $\left[\partial X_{m}^{\prime \prime} / \partial x_{i}\right]$, which is determined by multiplication (from left to right) of the three matrices given by Eqs. (A7), (A3), and (A1), respectively. Requiring the original ellipsoid central plane, $x_{3}=0$, to remain the ellipsoid central plane in the new coordinates, $X_{3}^{\prime \prime}=0$, we obtain

$\tan \chi=\epsilon \cot \theta$,

so that, to the first-order accuracy with respect $\epsilon$, we have

$$
\left[\frac{\partial X_{m}^{\prime \prime}}{\partial x_{i}}\right] \approx\left[\begin{array}{ccc}
\sin \theta \cos \varphi & \sin \theta \sin \varphi & 0 \\
-\sin \varphi & \cos \varphi & 0 \\
0 & 0 & \epsilon / \sin \theta
\end{array}\right]
$$

and

$\left[\frac{\partial x_{i}}{\partial X_{m}^{\prime \prime}}\right] \approx\left[\begin{array}{ccc}\cos \varphi / \sin \theta & -\sin \varphi & 0 \\ \sin \varphi / \sin \theta & \cos \varphi & 0 \\ 0 & 0 & \sin \theta / \epsilon\end{array}\right]$

Second, we rotate around the new $\boldsymbol{X}_{3}$-axis through an angle $\eta$ to the final coordinates, $X_{k}$,

$\left[\begin{array}{l}\partial X_{k} \\ \partial X_{m}^{\prime \prime}\end{array}\right]=\left[\begin{array}{ccc}\cos \eta & -\sin \eta & 0 \\ \sin \eta & \cos \eta & 0 \\ 0 & 0 & 1\end{array}\right]$

so that the entire transformation from $x_{i}$ to $X_{k}$ and back are determined by final Eqs. (8) and (9). Substituting $x_{i}$ in terms of $X_{k}$ into Eq. (1) and equating the factor in front of $X_{1} X_{2}$ to zero, to the first-order accuracy with respect to small parameters $\epsilon$ and $a_{3} / a_{1,2}$, we obtain for $\eta$ Eq. (10), which in turn results in Eqs. (12) and (13).

Now we transform Eqs. (A4) and (A5) to the final coordinates, $X_{k}$. Expressing the current density according to $J_{X_{k}}=\sum_{i=1}^{3}\left(\partial X_{k} / \partial X_{i}^{\prime}\right) J_{X_{i}^{\prime}}$ and transforming the electric field in Eq. (A4) according to $E_{X_{k}}^{\prime}=\sum_{i=1}^{3}\left(\partial X_{k}^{\prime} / \partial X_{i}\right) E_{X_{i}}$, we obtain

$J_{X_{i}}=\sum_{k=1}^{3} \sigma_{X_{k}}^{X_{i}} E_{X_{k}}$

with the final conductivity tensor

$\left[\sigma_{X_{k}}^{X_{i}}\right] \approx K \times\left[\begin{array}{ccc}1 & q \cos \chi & -p \sin \eta \\ -q \cos \chi & 1 & p \cos \eta \\ p \sin \eta & -p \cos \eta & 1\end{array}\right]$.

where $K$ and $q$ are defined by Eq. (A6), while

$p=q \sin \chi \approx\left(\frac{\psi}{1+\psi}\right)^{1 / 2} \cot \theta$.

When compared to Eq. (A5), Eq. (A12) contains additional off-diagonal Hall terms proportional to $p$, which originate 
from the final coordinate rotation. Besides, while the factors $\cos \chi$ in the large terms $\propto q \cos \chi$ are actually close to unity, we retain them anyway in order to ensure the invariance of $\operatorname{det}\left[\sigma_{X_{k}}^{X_{i}}\right]=\operatorname{det}\left[\begin{array}{c}X_{i}^{\prime} \\ \sigma_{X_{k}^{\prime}}^{\prime}\end{array}\right]=K^{3}\left(1+q^{2}\right)$. The antisymmetric off-diagonal terms in the RHS of Eq. (A12) do not contribute to the divergence of the current density, so that in the $X_{i}$-coordinates Eq. (3) becomes Eq. (15) and reduces to Laplace's Eq. (16).

\section{Appendix B}

\section{Extended cloud (meteor plasma trail)}

In this appendix, we briefly discuss the limit of an extended (rod-like) cloud, such as meteor plasma trails (Dimant and Oppenheim, 2006a). We studied the effect of an external field on the meteor trail in our previous paper (Dimant et al., 2009). In that paper, however, we employed a slightly different approach by assuming that along with the absence of internal currents in the $X_{3}$-direction there is also no significant internal current in the $X_{2}$-direction. In this paper, we have avoided such restriction. Using the general solution described above, we consider the extended-cloud limit of $a_{2,3} \ll a_{1}$ and compare the results with those of Dimant et al. (2009). This will allow us to obtain a limitation on the previously found solution. Being interested only in this issue, we restrict our discussion to the parameter dependence of the internal electric field.

In meteor trails, we have comparable sizes $a_{2}$ and $a_{3}, a_{3} \sim$ $a_{2} \ll a_{1}$. This provides automatically $A_{3} \ll A_{2} \ll A_{1}$, which fits our key restriction of Eq. (14). For the sake of simplicity, we consider here a meteor plasma trail directed strictly perpendicular to the magnetic field, $\theta=90^{\circ}$ with $\varphi=0$, so that $\chi, p, \eta=0, A_{1}=A_{2}=a, A_{3}=\epsilon a_{3}, E_{X_{1,2}}^{(0)}=E_{1,2}^{(0)}$, and, according to Eq. (32) from Dimant et al. (2009), we also have

$\Lambda_{1} \approx \ln \frac{4 A_{1}}{A_{2}}-1 \gtrsim 1, \quad \Lambda_{2} \approx \frac{A_{2}}{A_{1}}=\frac{a_{2}}{a_{1}} \ll 1$.

As a result, we obtain from Eqs. (34) and (35) $\gamma_{1,2}=q \alpha_{1,2}$ and

$$
\begin{aligned}
& \alpha_{1} \approx \frac{\Lambda_{1} A_{2} A_{3} n^{\mathrm{int}}}{A_{1}^{2} n_{0}}=\frac{\epsilon \Lambda_{1} a_{2} a_{3} n^{\mathrm{int}}}{a_{1}^{2} n_{0}}, \\
& \alpha_{2} \approx \frac{A_{3} n^{\mathrm{int}}}{A_{2} n_{0}}=\frac{\epsilon a_{3} n^{\mathrm{int}}}{a_{2} n_{0}} .
\end{aligned}
$$

For the internal electric field, we obtain from Eq. (38)

$$
\begin{aligned}
& E_{X_{1}}^{\mathrm{int}} \approx \frac{\left(1+\alpha_{2}\right) E_{X_{1}}^{(0)}-q \alpha_{1} E_{X_{2}}^{(0)}}{\left(1+\alpha_{1}\right)\left(1+\alpha_{2}\right)+q^{2} \alpha_{1} \alpha_{2}}, \\
& E_{X_{2}}^{\mathrm{int}} \approx \frac{\left(1+\alpha_{1}\right) E_{X_{2}}^{(0)}+q \alpha_{2} E_{X_{1}}^{(0)}}{\left(1+\alpha_{1}\right)\left(1+\alpha_{2}\right)+q^{2} \alpha_{1} \alpha_{2}} .
\end{aligned}
$$

In meteor trails, the parameters $\alpha_{1}$ and $\alpha_{2}$ usually differ by many orders of magnitude,

$\frac{\alpha_{2}}{\alpha_{1}} \approx \frac{a_{1}^{2}}{\Lambda_{1} a_{2}^{2}} \gg q^{2} \gg 1$,

so that Eq. (B2) further simplifies in two overlapping domains: (1) $\alpha_{2} \gg 1$, or

$1 \ll \frac{a_{2}}{\epsilon a_{3}} \ll \frac{n^{\mathrm{int}}}{n_{0}}$,

and (2) $\alpha_{1} \ll q^{2} \alpha_{1} \ll 1$, or

$\frac{n^{\mathrm{int}}}{n_{0}} \ll \frac{a_{1}^{2}}{\epsilon q^{2} \Lambda_{1} a_{2} a_{3}} \ll \frac{a_{1}^{2}}{\epsilon \Lambda_{1} a_{2} a_{3}}$.

In the domain (1), we obtain

$E_{X_{1}}^{\mathrm{int}} \approx \frac{E_{X_{1}}^{(0)}-\left(q \alpha_{1} / \alpha_{2}\right) E_{X_{2}}^{(0)}}{1+\left(q^{2}+1\right) \alpha_{1}} \approx \frac{E_{X_{1}}^{(0)}}{1+q^{2} \alpha_{1}}$,
$E_{X_{2}}^{\mathrm{int}} \approx \frac{\left[\left(1+\alpha_{1}\right) / \alpha_{2}\right] E_{X_{2}}^{(0)}+q E_{X_{1}}^{(0)}}{1+\left(q^{2}+1\right) \alpha_{1}} \approx \frac{q E_{X_{1}}^{(0)}}{1+q^{2} \alpha_{1}}$,

while in the domain (2) we obtain

$E_{X_{1}}^{\mathrm{int}} \approx E_{X_{1}}^{(0)}-\frac{q \alpha_{1} E_{X_{2}}^{(0)}}{1+\alpha_{2}} \approx E_{X_{1}}^{(0)}$,

$E_{X_{2}}^{\mathrm{int}} \approx \frac{E_{X_{2}}^{(0)}+q \alpha_{2} E_{X_{1}}^{(0)}}{1+\alpha_{2}}$

where we assumed comparable $E_{X_{1,2}}^{(0)}$ and took into account that, according to Eq. (B3), $q \alpha_{1} / \alpha_{2} \ll q^{-1} \ll 1$.

In domain (1), the field component along the major trail axis, $E_{X_{1}}^{(0)}$, is the only important external field component, while $E_{X_{2}}^{(0)}$ is inconsequential. Equation (B6) agrees with the results of Dimant et al. (2009), where the term $q^{2} \alpha_{1}$ in the denominators of Eq. (B6) corresponds (for $\theta=90^{\circ}$ ) to the parameter $k$ in Dimant et al. (2009). In this domain, we have $E_{X_{2}}^{\mathrm{int}} \approx q E_{X_{1}}^{\mathrm{int}} \gg E_{X_{1}}^{\mathrm{int}}$. The large internal field component perpendicular to $\boldsymbol{E}_{X_{1}}^{(0)}$ is due to trail polarization by the Hall current in the direction perpendicular to the external field (this effect is fully analogous to that in the electrojet formation). If the trail is sufficiently dense, $k=q^{2} \alpha_{1}$ $\gg 1$, i.e., if $n^{\text {int }} / n_{0} \gg a_{1}^{2} /\left(\epsilon q^{2} \Lambda_{1} a_{2} a_{3}\right)$, then the major field $\boldsymbol{E}_{X_{1}}^{(0)}$ penetrates into the trail only weakly, $E_{X_{1}}^{\text {int }} \ll E_{X_{1}}^{(0)}$. If, at the same time, $1 / q \ll q \alpha_{1}<1$, i.e., if $a_{1}^{2} /\left(\epsilon q^{2} \Lambda_{1} a_{2} a_{3}\right) \ll$ $n^{\text {int }} / n_{0}<a_{1}^{2} /\left(\epsilon q \Lambda_{1} a_{2} a_{3}\right)$ then the total internal electric field magnitude, $\left|\boldsymbol{E}_{\boldsymbol{X}}^{\text {int }}\right| \approx E_{X_{2}}^{\text {int }}$, remains larger than the external field magnitude. For a much denser trail, $q \alpha_{1} \gg 1, n^{\text {int }} / n_{0}$ $\gg a_{1}^{2} /\left(\epsilon q \Lambda_{1} a_{2} a_{3}\right)$, the total internal field is much less than the external field. 
Within domain (1), as the ratio $n^{\text {int }} / n_{0}$ decreases, $E_{X_{2}}^{\text {int }}$ reaches its maximum value, $q E_{X_{1}}^{(0)}$, at the lowest-density margin, which lies within a domain where domains (1) and (2) overlap, $q^{2} \alpha_{1} \ll 1$ and $\alpha_{2} \gg 1$, i.e., where $a_{2} / \epsilon a_{3} \ll n^{\text {int }} / n_{0}$ $\ll a_{1}^{2} /\left(\epsilon q^{2} \Lambda_{1} a_{2} a_{3}\right)$, and both Eqs. (B6) and (B7) are valid, $E_{X_{1}}^{\text {int }} \approx E_{X_{1}}^{(0)}$ and $E_{X_{2}}^{\text {int }} \approx q E_{X_{1}}^{(0)}$. As the density ratio $n^{\text {int }} / n_{0}$ decreases further we reach the point where $\alpha_{2}$ becomes $\lesssim 1\left(n^{\mathrm{int}} / n_{0} \lesssim a_{2} / \epsilon a_{3}\right)$, so that we leave the overlap domain but remain within domain (2). Here, the expression $E_{X_{1}}^{\text {int }} \approx E_{X_{1}}^{(0)}$ remains valid, while $E_{X_{2}}^{\text {int }}$ starts deviating from $q E_{X_{1}}^{(0)}$ until it approaches $E_{X_{2}}^{(0)}$ in the limit of $\alpha_{2} \ll 1$, i.e., $n^{\text {int }} / n_{0} \ll a_{2} /\left(q \epsilon a_{3}\right)$. This deviation from the results of Dimant et al. (2009) shows that their field of applicability is restricted by domain (1). In diffusing meteor trails, we usually have $a_{2} / a_{3} \simeq[\psi /(1+\psi)]^{1 / 2} \lesssim 1$ (Dimant and Oppenheim, 2006a, Appendix B). According to Eqs. (7), the condition of Eq. (B4) can be rewritten as

$$
\frac{n^{\text {int }}}{n_{0}} \gg \frac{\psi^{1 / 2}}{(1+\psi) \Theta_{0}} \simeq \frac{70 \psi^{1 / 2}}{1+\psi} .
$$

This is the sought-for limitation of the results obtained previously for meteor trails (Dimant et al., 2009). For actual meteor trails in the night-time ionosphere, it becomes essential during the later stage of the trail diffusion when the effects of external electric field become much less important.

Acknowledgements. This work was supported by National Science Foundation Ionospheric Physics Grants No. ATM-0819914, ATM0613624, ATM-0640609, and ATM-0442075. The authors thank V. G. Merkin for useful discussions of conductance issues in global MHD models.

Topical Editor M. Pinnock thanks two anonymous referees for their help in evaluating this paper.

\section{References}

Bristow, W. A. and Watkins, B. J.: Effect of the large-scale convection electric field structure on the formation of thin ionization layers at high latitudes, J. Atmos. Terr. Phys., 56, 401-415, 1994.

Cosgrove, R. B. and Tsunoda, R. T.: Polarization electric fields sustained by closed-current dynamo structures in midlatitude sporadic E, Geophys. Res. Lett., 28, 1455-1458, doi:10.1029/ 2000GL012178, 2001.

Cosgrove, R. B. and Tsunoda, R. T.: Wind-shear-driven, closedcurrent dynamos in midlatitude sporadic E, Geophys. Res. Lett., 29, 1020, doi:10.1029/2001GL013697, 2002a.

Cosgrove, R. B. and Tsunoda, R. T.: A direction-dependent instability of sporadic-E layers in the nighttime midlatitude ionosphere, Geophys. Res. Lett., 29, 1864, doi:10.1029/2002GL014669, 2002b.

Cosgrove, R. B. and Tsunoda, R. T.: Simulation of the nonlinear evolution of the sporadic-E layer instability in the nighttime midlatitude ionosphere, J. Geophys. Res., 108, 1283, doi: 10.1029/2002JA009728, 2003.
Cosgrove, R. B. and Tsunoda, R. T.: Instability of the E-F coupled nighttime midlatitude ionosphere, J. Geophys. Res., 109, A04305, doi:10.1029/2003JA010243, 2004.

Dimant, Y. S. and Oppenheim, M. M.: Meteor trail diffusion and fields: 1. Simulations, J. Geophys. Res., 111, A12312, doi:10. 1029/2006JA011797, 2006a.

Dimant, Y. S. and Oppenheim, M. M.: Meteor trail diffusion and fields: 2. Analytical theory, J. Geophys. Res., 111, A12313, doi: 10.1029/2006JA011798, 2006b.

Dimant, Y. S., Oppenheim, M. M., and Milikh, G. M.: Meteor plasma trails: effects of external electric field, Ann. Geophys., 27, 279-296, 2009,

http://www.ann-geophys.net/27/279/2009/.

Farley, D. T.: A theory of electrostatic fields in a horizontally stratified ionosphere subject to a vertical magnetic field, J. Geophys. Res., 64, 1225-1233, 1959.

Farley, D. T.: A theory of electrostatic fields in the ionosphere at nonpolar geomagnetic latitudes, J. Geophys. Res., 65, 869-877, 1960.

Fedder, J. A., Slinker, S. P., Lyon, J. G., and Elphinstone, R. D.: Global numerical simulation of the growth phase and the expansion onset for substorm observed by Viking, J. Geophys. Res., 100, 19083-19093, 1995.

Haldoupis, C., Schlegel, K., and Farley, D. T.: An explanation for type 1 radar echoes from the midlatitude E-region ionosphere, Geophys. Res. Lett., 101, 97-100, 1996.

Hysell, D. L. and Burcham, J. D.: The 30-MHz radar interferometer studies of midlatitude E region irregularities, J. Geophys. Res., 105, 12797-12812, doi:10.1029/1999JA000411, 2000.

Hysell, D. L., Yamamoto, M., and Fukao, S.: Simulations of plasma clouds in the midlatitude $\mathrm{E}$ region ionosphere with implications for type I and type II quasiperiodic echoes, J. Geophys. Res., 107, 1313, doi:10.1029/2002JA009291, 2002.

Kelley, M. C.: The Earth's Ionosphere, Academic, San Diego, California, 1989.

Kirkwood, S. and Nilsson, H.: High-latitude Sporadic-E and other Thin Layers - the Role of Magnetospheric Electric Fields, Space Sci. Rev., 91, 579-613, 2000.

Landau, L. D. and Lifshitz, E. M.: Electrodynamics of Continuous Media, Eddison-Wesley, Reading, MA, 1960.

Maruyama, T., Fukao, S., and Yamamoto, M.: A possible mechanism for echo striation generation of radar backscatter from midlatitude sporadic E, Radio Sci., 35, 1155-1164, doi:10.1029/ 1999RS002296, 2000.

Mathews, J. D.: Sporadic E: current views and recent progress, J. Atmos. Terr. Phys., 60, 413-435, 1998.

Merkin, V. G., Milikh, G., Papadopoulos, K., Lyon, J., Dimant, Y. S., Sharma, A. S., Goodrich, C., and Wiltberger, M.: Effect of anomalous electron heating on the transpolar potential in the LFM global MHD model, Geophys. Res. Lett., 32, L22101, doi: 10.1029/2005GL023315, 2005.

Moen, J. and Brekke, A.: The solar flux influence on quiet time conductances in the auroral ionosphere, Geophys. Res. Lett., 20, 971-974, doi:10.1029/92GL02109, 1993.

Ogawa, T., Yamamoto, M., and Fukao, S.: Middle and upper atmosphere radar observations of turbulence and movement of midlatitude sporadic E irregularities, J. Geophys. Res., 100, 12173 12188, 1995.

Ogawa, T., Takahashi, O., Otsuka, Y., Nozaki, K., Yamamoto, M., 
and Kita, K.: Simultaneous middle and upper atmosphere radar and ionospheric sounder observations of midlatitude E region irregularities and sporadic E layer, J. Geophys. Res., 107, 1275, doi:10.1029/2001JA900176, 2002.

Pan, C. J. and Tsunoda, R. T.: Quasi-periodic echoes observed with the Chung-Li VHF radar during the SEEK campaign, Geophys. Res. Lett., 25, 1809-1812, doi:10.1029/98GL00302, 1998.

Raeder, J., McPherron, R. L., Frank, L. A., Kokubun, S., Lu, G., Mukai, T., Paterson, W. R., Sigwarth, J. B., Singer, H. J., and Slavin, J. A.: Global simulation of the Geospace Environment Modeling substorm challenge event, J. Geophys. Res., 106, 381395, 2001.

Ridley, A. J., Gombosi, T. I., and DeZeeuw, D. L.: Ionospheric control of the magnetosphere: conductance, Ann. Geophys., 22, 567-584, 2004, http://www.ann-geophys.net/22/567/2004/.

Riley, K. F. and Hobson, M. P. and Bence, S. J.: Mathematical methods for physicists and engineers, Cambridge University Press, 1008 pp., 1998.

Rozhansky, V. A. and Tsendin, L. D.: Transport Phenomena in Partially Ionized Plasma, Taylor \& Francis, London-New York, 2001.

Shalimov, S. and Haldoupis, C.: E-region wind-driven electrical coupling of patchy sporadic- $E$ and spread- $F$ at midlatitude, Ann. Geophys., 23, 2095-2105, 2005, http://www.ann-geophys.net/23/2095/2005/.

Shalimov, S., Haldoupis, C., and Schlegel, K.: Large polarization electric fields associated with midlatitude sporadic E, J. Geophys. Res., 103, 11617-11626, 1998.
Tsunoda, R. T.: On polarized frontal structures, type-1 and quasiperiodic echoes in midlatitude sporadic E, Geophys. Res. Lett., 25, 2641-2644, doi:10.1029/98GL01934, 1998.

Tsunoda, R. T.: On the coupling of layer instabilities in the nighttime midlatitude ionosphere, J. Geophys. Res., 111, A11304, doi: 10.1029/2006JA011630, 2006.

Tsunoda, R. T. and Cosgrove, R. B.: Coupled electrodynamics in the nighttime midlatitude ionosphere, Geophys. Res. Lett., 28, 4171-4174, doi:10.1029/2001GL013245, 2001.

Tsunoda, R. T., Cosgrove, R. B., and Ogawa, T.: Azimuthdependent Es layer instability: A missing link found, J. Geophys. Res., 109, A12303, doi:10.1029/2004JA010597, 2004.

Whitehead, J. D.: Production and Prediction of Sporadic E, Rev. Geophys. Space Phys., 8, 65-144, 1970.

Whitehead, J. D.: Recent work on mid-latitude and equatorial sporadic-E, J. Atmos. Terr. Phys., 51, 401-424, 1989.

Yamamoto, M., Fukao, S., Tsuda, T., Kato, S., Woodman, R. F., and Ogawa, T.: Mid-latitude E region field-aligned irregularities observed with the MU radar, J. Geophys. Res., 96, 15943-15949, 1991.

Yokoyama, T., Yamamoto, M., and Fukao, S.: Computer simulation of polarization electric fields as a source of midlatitude fieldaligned irregularities, J. Geophys. Res. (Space Physics), 108, 1054, doi:10.1029/2002JA009513, 2003.

Yokoyama, T., Yamamoto, M., Fukao, S., and Cosgrove, R. B.: Three-dimensional simulation on generation of polarization electric field in the midlatitude E-region ionosphere, J. Geophys. Res., 109, A01309, doi:10.1029/2003JA010238, 2004. 